\title{
Delayed multiattribute product differentiation is
}

\author{
Thomas A. Weber* \\ Department of Management Science and Engineering, Terman Engineering Center, Stanford University, Stanford, CA 94305-4026, USA
}

Received 24 January 2007; received in revised form 17 April 2007; accepted 9 June 2007

Available online 20 June 2007

\begin{abstract}
We develop a two-stage model for versioning products with respect to both vertical and horizontal attributes. At first, a firm positions its top-quality "flagship" product in a market with an imperfectly known distribution of tastes and reservation prices. In the second stage, the firm learns these consumer characteristics and has the option of extending its product line by versioning the flagship product using pure horizontal differentiation, quality degrading, or both. The firm's nonconvex versioning problem is solved analytically for the two-product case. We find that ex ante extending the product line through vertical differentiation is optimal for low marginal cost of quality (development cost); otherwise pure horizontal differentiation is superior. Given quasilinear consumer preferences and a uniform distribution of consumer characteristics, versioning with respect to both horizontal and vertical attributes is never optimal. Under delayed differentiation the optimal policy is contingent on the observed demand realization and may lead to horizontal cannibalization and price dispersion for equal-quality products. The firm tends to increase its investment in product quality unless it adopts a state-contingent policy of horizontal versioning for high and vertical versioning for low demand realizations. Following a state-contingent policy, the optimal upfront development effort may be significantly lower than under full ex-ante commitment. The option value of delayed differentiation is generally nonmonotonic in the firm's development cost.

(C) 2007 Elsevier B.V. All rights reserved.
\end{abstract}

Keywords: Multiproduct firms; Product versioning; Delayed differentiation

\footnotetext{
is This research was in part supported by a David Morgenthaler II Faculty Scholar Award at Stanford University. I would like to thank Krishnan Anand, Jianqing Chen, Eric Clemons, David Croson, Rachel Croson, Lorin Hitt, Richard Kihlstrom, Paul Kleindorfer, Karl Ulrich, Andrew Whinston, two anonymous referees, as well as participants of the 2001 International Conference on Information Systems (ICIS) in New Orleans, the 2002 INFORMS Annual Meeting, and seminars at the Wharton School and Carnegie Mellon University for helpful comments and suggestions. An earlier version of this paper was nominated for the 2001 ICIS Best Research Paper Award. Any remaining errors are my own.

* Tel.: +1 650725 6827; fax: +1 6507231614 .

E-mail address: webert@stanford.edu.
}

\section{Introduction}

Information goods such as computer software or electronic newspapers can be provided by firms at a low marginal cost, though in many cases large capital outlays are required to produce their first unit. The substantial setup cost is driven mainly by the cost of developing the top quality product. Having established this "flagship" product, a firm can degrade it or in other ways modify it, and in this way create a multitude of products with different attributes at a small "versioning" cost. Information goods are not the only products that enjoy such cost complementarities in development. Component standardization and design based on common platforms can be found in a wide range of industries [35]. In 
addition, information goods are often bundled in with physical goods, determining in part the functionality of these underlying physical products. Therefore, finding optimal versioning policies is becoming increasingly important, as often lower distribution costs and newfound customer intimacy render intricate second-degree price discrimination strategies feasible. We present a model of a firm that chooses a two-product portfolio sequentially, when products can differ with respect to both horizontal and vertical nonprice attributes. The firm determines its second, or versioned product, after it has completed the development of its flagship product, and uncertainty over the market acceptance of the latter has resolved.

It is well known that component sharing or, in a broader sense, cost complementarities between products can induce a firm to increase its product variety by keeping the number of components necessary for their assembly manageable [12]. On the other hand, over time the creation of product variety should be commensurate with the information available about demand, leading to the practice of delayed differentiation $[1,21,33]$. Cost complementarities and opportunities for delayed differentiation are especially large for information goods: provided a sufficiently modular product design, features can be easily disabled or rebundled, leading to vertically and horizontally differentiated versions of the initial flagship product [32]. Since most of the development effort goes into the design of the top quality product, the optimal initial research effort and therefore ultimately the choice of the flagship product's quality level depends on the firm's options for creating a versioned product once the market demand for the first product has been observed. ${ }^{1}$ While this paper is not so much concerned with the timing of product introductions [24], we are interested in finding an optimal segmentation of a heterogeneous customer base of multiple characteristics both with and without delayed differentiation. We derive the option value for delaying the versioning decision and show that investment in product quality does not have to be higher when this option is available.

All consumers are initially endowed with a budget and personal taste. Each consumer of a certain (taste, budget)type has a utility, which depends on price, product quality, and distance of the horizontal product attribute

\footnotetext{
${ }^{1}$ As an example, Microsoft's Office 2000 software was available in at least four different versions (Premium, Professional, Small Business, and Standard) essentially to segment the market and screen the heterogeneous consumer base. These versions contained both vertically (i.e., more $v$ s. fewer features) and horizontally (i.e., disjoint features) differentiating attributes.
}

from one's own taste. Quality is a vertically differentiating instrument and can be thought of as performance or product breadth. If a product is characterized as a bundle of features [20], then a product including more features than another is of higher quality, whereas two products with different bundles of features, not distinguished in terms of performance or overall product breadth, can be seen as horizontally differentiated. Each of them appeals to consumer tastes "located" sufficiently close. Given a distribution of consumer types (here assumed to be uniform for simplicity), generated for instance by usage preferences or existing standards, the firm can choose product attributes so as to attract as many of the consumers with a sufficiently large budget (i.e., potential buyers) as possible. More specifically, the goal of the risk-neutral firm is to create a certain number of product offerings of the form (price, quality, horizontal product attribute), such that its expected profits are maximized. Each product offering targets a subset or segment of the (taste,budget)-space, into which the consumers self-select by choosing the product that maximizes their respective utilities. However, at the outset the firm's ability to optimize the choice of its portfolio is limited by imperfect information about consumer characteristics. We assume that this subjective uncertainty resolves after the creation of its flagship product, which typically can be used to evaluate demand. This creates an option value of delaying differentiation to reduce the firm's partially irreversible commitment to a particular product portfolio.

The underlying problem of multiattribute product differentiation is highly nonconvex. We show that both pure horizontal and pure vertical versioning are locally optimal, whereas mixed versioning, i.e., the simultaneous differentiation along both horizontal and vertical nonprice attributes, is ex ante never optimal in our quasilinear homogeneous setting. Pure vertical versioning is globally optimal for relatively low development costs, whereas for high development costs pure horizontal versioning is superior. Under delayed differentiation the optimal policy is contingent on the demand realization ("state"). A consequence of this added flexibility may be that the firm's optimal ex-ante investment in product development may drastically drop, instead of increase as a result of diminished (strategic) irreversibility of the upfront sunk cost.

\subsection{Literature review}

Beginning with Hotelling's seminal paper on horizontal competition [15], numerous contributions have been made to product differentiation. The corresponding literature can be divided into locational models in the 
tradition of Hotelling, where each firm is given an "address" in product space, and so-called "non-address" models in the spirit of Chamberlin's monopolistic competition [8], where a representative consumer exhibits (probabilistic) preferences for different products. $^{2}$ An important distinction between the two groups of models is that in the latter group, each product is competing with all others, while in the former consumers are truly heterogeneous in their preferences, and some products may have no overlap, i.e., may never be in direct competition. ${ }^{3}$ In this paper we adopt the locational approach, which in our view better captures consumer heterogeneity and allows the explicit consideration of participation constraints that inevitably arise when dealing with a spatial distribution of endowed unobservable consumer characteristics. Our model is inspired by Salop's "circular city" [30], which we extend to a "cylinder" by adding a vertical product characteristic.

Mussa and Rosen [25], based on earlier work by Mirrlees [23], started a line of work on second-degree price discrimination of multiproduct firms with quality as the differentiating instrument. Cremer and Thisse [10] demonstrate that in many setups horizontal differentiation can be seen as a special case of vertical differentiation. However, as our model contains both horizontal and vertical features, it cannot be generally mapped into either a pure horizontal or a pure vertical one, as we shall see below. Jones and Mendelson [17] show that for quality-differentiated information goods and a uniform distribution of consumer types, no differentiation is optimal. ${ }^{4}$ Our results do not confirm these findings. Interpreting one consumer characteristic as reservation price induces a type-dependent participation (or "feasibility") constraint: no consumer can pay more than her reservation price. This partitions the consumer base into segments buying different products.

In addition to allowing the multidimensional screening of a heterogeneous consumer base [19,29], product variety and broader product lines based on a modular product design ${ }^{5}$ can help to delay differentiation along

\footnotetext{
${ }^{2}$ For a good bibliography see Refs. [2,4,34].

3 A notable exception in this dichotomy is the model in Ref. [27] that combines characteristics from both locational and non-address models, driven by symmetry assumptions in the preferences of a representative consumer who is faced with localized products.

${ }^{4}$ These results have been generalized by Ref. [6], which provides sufficient conditions for goods with nonzero marginal costs and general utility functions under which a stable, incentive-compatible separation of the consumer base into segments can be reached.

${ }^{5}$ Ref. [18] provides an excellent survey of the recent literature on product development decisions.
}

the supply chain $[21,33]$. The real-option value of delaying irreversible commitment to a full product line can be seen in analogy to financial decision making [22]: delaying irreversible investments can carry a significant value, sometimes comparable to the investment volume itself [28]. This option value is generally increasing in the magnitude of the uncertainty unless, as Ref. [16] shows, imperfectly correlated risks from different sources are pooled, potentially decreasing the value of managerial flexibility. We restrict ourselves to what [16] terms "market payoff variability," a single risk class, and thus circumvent this effect. Examining the realoption value of delaying the product versioning until with release of the flagship product - overall demand has been observed, we find that it is generally nonmonotonic in the cost of quality $\beta$. Naturally, the option value depends in an important manner on the performance of the default ex-ante versioning policy, and is therefore not necessarily monotonic in the amount of uncertainty over all $\beta$ 's. This points to resource flexibility as a driver of this option value [36]. Delaying differentiation does not generally allow one to reverse the upfront quality investment. Being able to make product-line-extension and pricing decisions contingent on the observed demand carries significant value depending on the firm's default policy. For instance for development costs, $\beta$, close to zero when the irreversibility of the investment in flagship quality is not very significant, the option to delay differentiation carries a high value. In fact we show that it is locally maximal at $\beta=0$. The effects of increased versioning flexibility on upfront investment in product development as well as on consumer surplus are ambiguous. We provide an example where delayed differentiation leads to a lower flagship quality as a result of a statecontingent policy where the mode of differentiation depends on the observed demand realization, which is generally the case if the dispersion in the firm's prior beliefs about demand is large. For very little demand uncertainty, the versioning mode is generally "locked in" ex ante, and the delay in the differentiation allows slight improvements on the previous pricing and positioning decisions. Consumer surplus depends both on the actual product development costs as well as on the chosen mode of product differentiation, and thus may vary in both directions when introducing an option to delay the versioning decision. To the best of our knowledge this paper, based on Ref. [37], provides a first complete analytical treatment of multiattribute versioning combining horizontal and vertical differentiation. We further extend this new approach to account for demand uncertainty by deriving optimal state- 


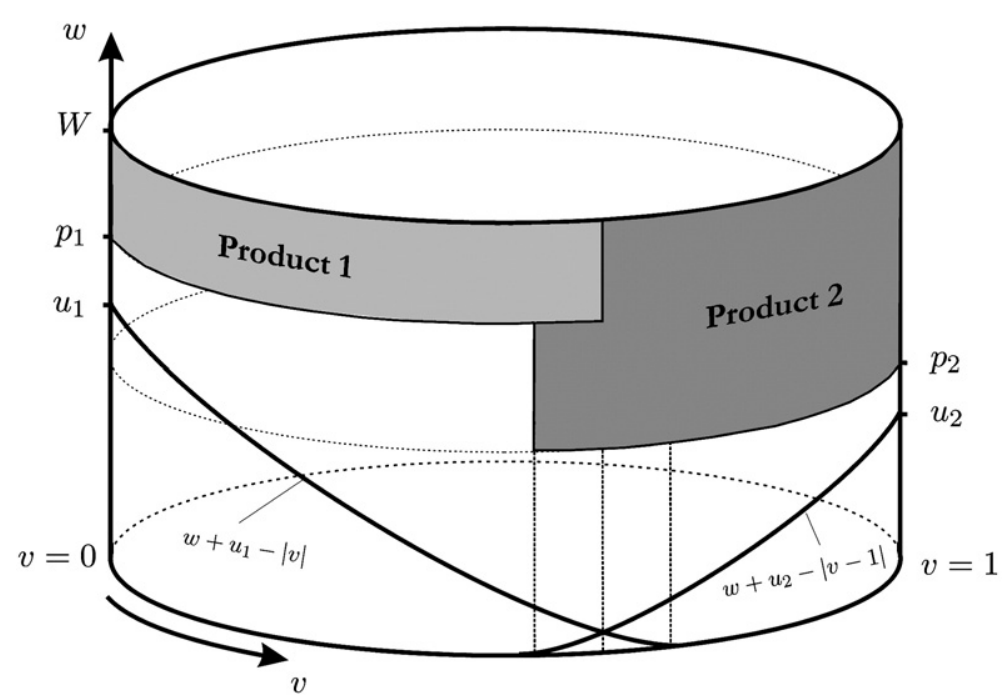

Fig. 1. Consumer utilities and resulting market segments $\left(q_{1}>q_{2}\right)$.

contingent policies under delayed differentiation. Information goods are chosen here for mere convenience. Everything in this paper equally applies to goods with positive marginal costs ( $c f$. Section 4.4).

\subsection{Outline}

In the following section we introduce the basic model together with some structural simplifications that can be made without loss of any generality. In Section 3 we examine the ex-ante versioning case in which both flagship and versioned product are chosen simultaneously. We show that mixed versioning is never optimal and that investment in quality increases with estimated demand. Section 4 covers the case where the firm possesses the option to delay product versioning until after demand has realized. We determine the optimal versioning policy and compute the option value of delayed differentiation in this context. We also show that investment in product quality under delayed differentiation may either increase or decrease using a state-contingent horizontal-vertical versioning policy. Section 5 discusses the results and concludes with directions for further research.

\section{The model}

Let all consumers be distributed uniformly on $\mathcal{V} \times \mathcal{W}$, where $\mathcal{V}=\mathbb{R} / 2 \mathbb{Z}$ and $\mathcal{W}=[0, W]$ denote the sets of tastes and budget levels, $W>0$ the maximum reservation price, and $\mathbb{R}, \mathbb{Z}$ the sets of reals and integers. The (quotient) space $\mathbb{R} / 2 \mathbb{Z}$ corresponds to an interval $[0,2]$ with the points 0 and 2 identified, so that $\mathcal{V} \times \mathcal{W}$ is a cylinder of radius $1 / \pi$ and of height $W(c f$.
Fig. 1). ${ }^{6}$ The parameter $W$ represents the maximum reservation price and is initially only imperfectly known, in terms of a bounded measurable probability density function $f$ with compact support $\mathcal{S} \subset \mathbb{R}_{++}$. We assume that any information product offered can be uniquely described in terms of its attributes, price $p$, nonnegative quality $q$, and taste $z \in \mathcal{V}$. The utility of a consumer of type $(v, w) \in \mathcal{V} \times \mathcal{W}$ who buys a product of attributes $(p$, $q, z)$ is assumed to be additively separable of the form $w-p+\kappa q-|v-z|$, and equal to $w$ if she does not buy the product. $^{7}$ The positive constant $\kappa$ defines the marginal valuation of quality relative to the other product attributes, price and taste. ${ }^{8}$ This consumer can only afford the product if her reservation price (disposable budget) $w$ is at least equal to the price $p$ of the product,

$p \leq w$.

\footnotetext{
${ }^{6}$ This model generalizes the common setup of "circular city" models in the spirit of Ref. [30], which are used to describe pure horizontal differentiation, typically to avoid the endpoint effects in Hotelling's "linear city." We have chosen the cylindrical setup for the mixed-differentiation model with precisely this in mind.

${ }^{7}$ General conditions for the additive separability of utility functions in budget have been provided by Ref. [14]. Instead of $\kappa q$ it is possible to incorporate any nonlinear strictly increasing quality measure $\phi(q)$ which simply corresponds to a rescaling ( $c f$. also Eq. (4)).

${ }^{8}$ Without loss of generality the marginal utility of taste differences $|v-z|$ can be normalized to one, even when the cylinder circumference $2 V$ (for some $V>0$ ) is not precisely known. Varying $V$ changes the relative marginal valuation of taste mismatch. By substituting $W^{\prime}=W / V$ for $W$ and using an appropriate prior probability density $g$ of $W^{\prime}$ (instead of $f$ ) one obtains the same results as the ones derived for the unit cylinder $\mathcal{V} \times \mathcal{W}$, up to a constant factor. Thus, the results in this paper do also hold when both maximum reservation price and extent of the taste domain are ex-ante uncertain.
} 
For any given maximum reservation price $W$ the normalization constant for the consumer density is $1 /(2 W)$. The total budget in the set $\mathcal{V} \times \mathcal{W}$ is $W / 2$, independent of the diameter of the cylinder.

\subsection{Change of variables}

To simplify some of the discussions that follow, we introduce the new variable $u=\kappa q-p$, so that consumer utility can be written in the form $w+u-|v-z|$. The new variable $u$ represents the net utility gain at perfect horizontal fit. Using this simple change of variables, consumer utility becomes independent of price. The participation constraint (i.e., for any consumer the utility obtained for the product has to weakly exceed utility for budget $w$ ) can be written as

$|v-z| \leq u$.

Any optimization of the product portfolio at the firm level needs to account for the feasibility and participation constraints (1)-(2) as well as incentive compatibility in the sense that each consumer's choice is utility maximizing.

\subsection{Cost structure}

Throughout most of this paper we consider information goods with zero marginal cost, which simplifies the closed-form solutions. This assumption is not crucial and all results can be easily generalized to goods with positive marginal cost, as is shown in Section 4.4. For information goods, the costs of reproduction and distribution are indeed very small, so that this has become a standard assumption in much of the extant literature. ${ }^{9}$ The cost $\beta q$ of creating the flagship product depends linearly on the quality $q$, and can be written in the new variables as

$C(p, u)=\frac{\beta}{\kappa}(p+u)$,

where $\beta$ is a positive constant. Without loss of generality it is possible to set

$\kappa=1$,

as the results for any arbitrary value of $\kappa>0$ can be recovered by substituting everywhere $\beta^{\prime}=\beta / \kappa$ for $\beta$. The weight $\kappa$ in the utility function has the sole effect of scaling the firm's quality cost. In everything that follows we therefore assume that Eq. (4) holds.

\footnotetext{
${ }^{9}$ Information goods are chosen here merely to keep analytical complexity as low as possible. Of course, there are settings for which the small, but nonzero, nature of the marginal costs matters, such as in deriving asymptotic properties of large bundles of information goods $[3,13]$.
}

\subsection{Versioning problem}

After having created the flagship product, the riskneutral firm has the option to create a versioned product by varying product attributes in the following admissible ways:

(a) horizontal version (i.e., same quality, different taste) at a cost $\alpha_{\mathrm{H}} \geq 0$

(b) vertical version (i.e., lower quality, same taste) at a $\operatorname{cost} \alpha_{\mathrm{V}} \geq 0$,

(c) mixed version (i.e., lower quality, different taste) at a cost $\alpha_{\mathrm{M}} \in\left[\max \left\{\alpha_{\mathrm{H}}, \alpha_{\mathrm{V}}\right\}, \alpha_{\mathrm{H}}+\alpha_{\mathrm{V}}\right]^{10}$

This cost structure exhibits strong cost complementarities or economies of scope [26], as development costs are incurred only once to create the flagship product. ${ }^{11}$ The firm maximizes profits subject to the consumers' choice and participation. Solutions to this profitmaximization problem exhibit translation invariance: profits will not change by just horizontally translating all products, as a direct consequence of the circular symmetry. One can therefore arbitrarily fix the horizontal location of the first product to zero. Any implied solution then naturally represents an entire equivalence class of solutions to the more general problem with arbitrary horizontal location of the first product.

\subsection{Demand estimation}

As has been pointed out above, the demand parameter $W$ is initially only imperfectly known to the firm (cf. also footnote 8). For any product of price $p$ the profit function depends on $W$ through the marginal revenue density $p(W-p) /(2 W)$. By replacing the random value $W$ with a nonlinear estimator $\hat{W}=(E[1 / W])^{-1}$ we obtain the expected marginal revenue density. The following technical lemma will be used throughout the paper without special mention.

Lemma 1. (i) $E[p(W-p) /(2 W)]=p \quad(\hat{W}-p) /(2 \hat{W})$. (ii) $\hat{W}<\bar{W}$, where $\bar{W}=E(W)$.

The first part of this result is trivial but useful, as it allows replacing the imperfectly known $W$ with the nonrandom $\hat{W}$ when computing expected profits. Part (ii) indicates that when maximizing expected profits, uncertainty in $W$ prompts the firm to use as decision-

\footnotetext{
${ }^{10}$ This condition can also be written in terms of the $r$-norm of the row vector $\left(\alpha_{\mathrm{H}}, \alpha_{\mathrm{V}}\right)$, i.e., $\alpha_{\mathrm{M}}=\left\|\left(\alpha_{\mathrm{H}}, \alpha_{\mathrm{V}}\right)\right\|_{r}=\left(\alpha_{\mathrm{H}}^{r}+\alpha_{\mathrm{V}}^{r}\right)^{1 / r}$ for $r \in[1, \infty)$ and $\alpha_{\mathrm{M}}=\left\|\left(\alpha_{\mathrm{H}}, \alpha_{\mathrm{V}}\right)\right\|_{\infty}=\max \left\{\alpha_{\mathrm{H}}, \alpha_{\mathrm{V}}\right\}$ for $r=\infty$.

${ }_{11}$ A multiproduct cost structure of this form has also been proposed by Ref. [31].
} 
relevant "certainty equivalent" of $W$ an estimator $\hat{W}$ that is always strictly (and sometimes substantially) lower than the expected maximum reservation price $\hat{W} .^{12}$ Our demand specification for the single-product firm corresponds to a linear demand curve

$D(p, u)=(1-(p / W)) \min \{1, u\}$

with random intercept at $W>0$. The demand elasticity $p /(W-p)$ is independent of the consumers' net utility $u>0$. The demand specification considered here is somewhat nonstandard, since the consumer heterogeneity is with respect to their horizontal location and their income, while much of the extant literature has emphasized consumer heterogeneity with respect to horizontal location and marginal valuation of product quality.

\section{Ex-ante versioning}

\subsection{Benchmark: Single-product firm}

Before solving the multiproduct case we briefly discuss the single-product monopoly as a benchmark. Without loss of generality we can set $z_{1}=0$, in view of the translation invariance discussed above. With this the expected single-product monopoly profit $\bar{\Pi}_{1}\left(p_{1}, u_{1}\right)=$ $E\left[\Pi_{1}\left(p_{1}, u_{1} ; W\right)\right]$ as a function of $\left(p_{1}, u_{1}\right)$ becomes

$\bar{\Pi}_{1}\left(p_{1}, u_{1}\right)=\frac{p_{1}\left(\hat{W}-p_{1}\right)}{\hat{W}} \min \left\{u_{1}, 1\right\}-\beta\left(p_{1}+u_{1}\right)$.

It is clear that $u_{1}^{*} \leq 1$, since $\partial_{u_{1}} \bar{\Pi}\left(p_{1}, u_{1}\right)<0$ for any $\left(p_{1}, u_{1}\right) \in(0, \hat{W}) \times(1, \infty)$. By virtue of the linearity of the profit function in $u_{1}^{*} \in[0,1]$ maximum profits are achieved for $u_{1}^{*} \in\{0,1\}$, so that

$\left(p_{1}^{*}, u_{1}^{*}\right)= \begin{cases}(\hat{W}(1-\beta) / 2,1), & \text { if } \beta \leq 1, \\ (0,0), & \text { if } \beta>1 .\end{cases}$

Thus, the single-product firm would like to achieve "full horizontal market coverage" (i.e., $q_{1}^{*}=1+p_{1}^{*}$ ), whenever it decides to enter the market. ${ }^{13}$

Proposition 1. (i) The optimal expected profit of the single-product firm is

$\bar{\Pi}_{1}^{*}=\frac{\hat{W}}{4}(1-\beta)^{2}-\beta$,

\footnotetext{
${ }^{12}$ The relative difference between $\bar{W}$ and $\hat{W}$ can become arbitrarily large, as can the relative difference in the corresponding profits (when using $\bar{W}$ instead of $\hat{W}$ ). As an example, consider a uniform distribution of $W$ on an interval $[\varepsilon, 2-\varepsilon]$ with $0<\varepsilon<1$. Then $\bar{W}=1$ for all $\varepsilon \in(0,1)$, while $\hat{W}$ goes to zero as $\varepsilon \rightarrow 0^{+}$.

13 This result is driven by the linearity of the cost function. In the case where the cost function is strictly convex, say, of the form $\beta q^{2}$, there may exist interior solutions, making it impossible to derive closed-form solutions in the multiproduct case.
}

increasing in $\hat{W}$ and decreasing in $\beta$. (ii) The singleproduct firm enters the market if and only if

$\hat{W}>\frac{4 \beta}{(1-\beta)^{2}}$.

Relation (7) is a viability condition that imposes a lower bound on the maximum-reservation-price estimator $\hat{W}$ as a function of $\beta$. In what follows we will use the optimal expected single-product monopoly profits as a benchmark for the performance of the various differentiation modes in a multiproduct setting.

\subsection{Ex-ante product differentiation}

Due to the symmetry of the problem it is possible, without any loss in generality, possible to set $z_{1}=0$ and to restrict $z_{2}$ to the interval $[0,1]$ (otherwise just switch products and translate). Any solution satisfying these constraints defines an equivalence class of solutions to the versioning problem. There is one further simplification, which directly results from the linearity of the problem in $z_{2}$. Indeed, the optimal value of $z_{2}$ must be at an extremity of $[0,1]$, i.e., $z_{1}^{*} \in\{0,1\}$. The induced two canonical cases of vertical and horizontal differentiation have to be distinguished in the solution to the versioning problem. Furthermore we assume that the quality of the second product does not exceed the quality of the first product $\left(q_{1} \geq q_{2}\right)$, which after a linear variable transformation $(u=\kappa q-p)$ can be equivalently written in the form

$q_{1}=p_{1}+u_{1} \geq p_{2}+u_{2}=q_{2}$.

This assumption expresses the fact that in order for a "versioning" policy to be meaningful, the first (flagship) product has to be of higher quality than the derived product. Fig. 2 provides an overview of the various modes of differentiation that can arise: (1) no differentiation $\left(z_{2}=0, q_{1}=q_{2}\right)$, which is de facto equivalent to the single-product monopoly; (2) pure vertical differentiation $\left(z_{2}=0, q_{1}>q_{2}\right)$; (3) mixed differentiation $\left(z_{2}=1\right.$, $\left.q_{1}>q_{2}\right)$; and, (4) pure horizontal differentiation $\left(z_{2}=1\right.$, $q_{1}=q_{2}$ ). To simplify the exposition, we will first set versioning costs to zero, as their effect can be analyzed separately once all the main expressions for the zeroversioning cost base case have been established.

\subsubsection{Pure vertical differentiation}

The profit function $\bar{\Pi}_{\mathrm{V}}$ is linear in $u_{1}$ and $u_{2}$, so that we can concentrate on the situation when $u_{1}=u_{2}=1$, since otherwise the firm would only enter with one 


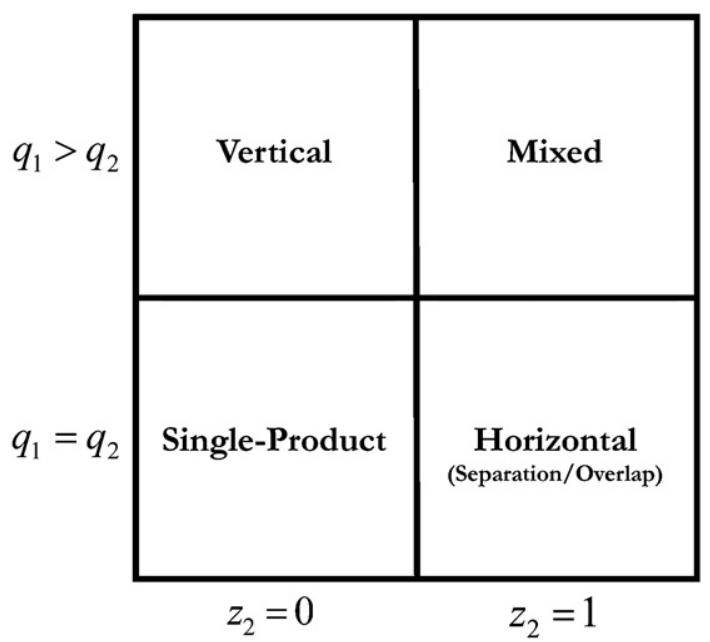

Fig. 2. Modes of differentiation.

product $\left(u_{2}=0\right.$, cf. Section 3.1). The maximization problem can be written in the form

$$
\begin{aligned}
& \max _{p_{1}, p_{2}} \bar{\Pi}_{\mathrm{V}}\left(p_{1}, p_{2}\right) \\
& \quad=\max _{p_{1}, p_{2}}\left\{\frac{1}{\hat{W}}\left(p_{1}\left(\hat{W}-p_{1}\right)+p_{2}\left(p_{1}-p_{2}\right)\right)-\beta\left(p_{1}+1\right)\right\},
\end{aligned}
$$

the solution of which yields the unique interior maximizers, $p_{1}^{*}=2 \hat{W}(1-\beta) / 3$ and $p_{1}^{*}=\hat{W}(1-\beta) / 3$, provided that $\beta<1$. Optimal expected profits are then

$\bar{\Pi}_{\mathrm{V}}^{*}=\frac{\hat{W}}{3}(1-\beta)^{2}-\beta=\bar{\Pi}_{1}^{*}+\frac{\hat{W}}{12}(1-\beta)^{2}$,

strictly greater than single-product monopoly profits $\bar{\Pi}_{1}^{*}$. Thus, pure vertical differentiation strictly dominates a single-product monopoly, given that versioning $\operatorname{costs} \alpha_{\mathrm{V}}$ are small enough. Pure vertical differentiation is ex-ante viable if and only if

$\hat{W}>\frac{3 \beta}{(1-\beta)^{2}}$.

It is important to note that since $u_{1}^{*}=u_{2}^{*}$ at the optimum in pure vertical differentiation, a consumer of type $(v, w)$ obtains equal surplus from both products. This follows from the latent assumption that in equilibrium consumers buy the higher-price product if this yields exactly the same utility as the lower-price product (e.g., since resale value is higher also). If this were not the case, the firm could technically achieve stable separation by letting $u_{1}^{*}=u_{1}^{*}+\varepsilon$ for any (arbi- trarily small) $\varepsilon>0$ without, in the limit, changing any of the results. The fundamental reason for the stable segmentation of the consumer population lies in the feasibility constraint (1): consumers never spend more than their budget $\mathrm{w}$ (reservation price) permits.

\subsubsection{Mixed differentiation}

In analogy to the single-product monopoly, profits $\bar{\Pi}_{\mathrm{M}}$ only depend linearly on $u_{1}$ and $u_{2}$ (implying full horizontal coverage). Since the constraint (8) is by assumption not binding, it is $u_{1}=u_{2}=1$. The profitmaximization problem thus takes the form

$$
\begin{aligned}
\max _{p_{1}, p_{2}} \bar{\Pi}_{\mathrm{M}}\left(p_{1}, p_{2}\right)= & \max _{p_{1}, p_{2}}\left\{\frac { 1 } { 2 \hat { W } } \left(p_{1}\left(\hat{W}-p_{1}\right)+p_{2}\left(\hat{W}-p_{2}\right)\right.\right. \\
& \left.\left.+p_{2}\left(p_{1}-p_{2}\right)\right)-\beta\left(p_{1}+1\right)\right\} .
\end{aligned}
$$

Straightforward unconstrained maximization yields the optimal prices $p_{1}^{*}=\hat{W}(5-8 \beta) / 7$ and $p_{2}^{*}=\hat{W}(3-2 \beta) /$ 7 , which are well defined (i.e., in $[0, \hat{W}]$ and such that $p_{1}^{*}>p_{2}^{*}$ ) for any positive $\beta<1 / 3$. Optimal expected profits are

$$
\bar{\Pi}_{\mathrm{M}}^{*}=\frac{\hat{W}}{7}\left(\frac{5}{4}-2 \beta\right)^{2}+\frac{\hat{W}}{16}-\beta=\bar{\Pi}_{1}^{*}+\frac{9 \hat{W}}{28}\left(\frac{1}{3}-\beta\right)^{2},
$$

strictly increasing in $\hat{W}$ and strictly decreasing in $\beta$. In the mixed-differentiation mode, the multi-product monopolist enters if and only if

$$
\hat{W}>\frac{\beta}{\frac{1}{7}\left(\frac{5}{4}-2 \beta\right)^{2}+\frac{1}{16}} \text {, and } \beta<\frac{1}{3} .
$$

Given entry in mixed-differentiation mode, quality dispersion does in general occur, since by construction for $\beta<1 / 3$ that $q_{1}^{*}=\hat{W}(5-8 \beta) / 7+1 \geq \hat{W}(3-2 \beta) / 7+1=q_{2}^{*}$. However, vertical differentiation strictly dominates mixed differentiation. Indeed,

$$
\begin{aligned}
\bar{\Pi}_{\mathrm{V}}^{*}-\bar{\Pi}_{\mathrm{M}}^{*} & =\hat{W}\left(\frac{(1-\beta)^{2}}{3}-\frac{1}{7}\left(\frac{5}{4}-2 \beta\right)^{2}-\frac{1}{16}\right) \\
& =\hat{W}\left(\frac{1}{20}-\frac{5}{21}\left(\beta-\frac{1}{10}\right)^{2}\right)>0
\end{aligned}
$$


for all $\beta \in(0,1 / 3)$. Therefore, the mixed-differentiation mode does not play a role, which by itself is an interesting fact.

Proposition 2. For any given viable market configuration $(\beta, \hat{W})$ either pure horizontal or pure vertical differentiation is optimal.

Mixed versioning is dominated by pure vertical differentiation. As soon as goods are horizontally differentiated with $\left(z_{2}=1\right)$, it is best to reduce the quality of the flagship product so as to economize on providing costly horizontal coverage with each product. Hence, leaving slack in constraint (8) does not fully utilize the potential of the flagship product, whose quality will ex ante be reduced until $q_{1}=q_{2}$, i.e., until pure horizontal differentiation is obtained.

\subsection{Pure horizontal differentiation}

In this mode constraint (8) is binding, so that $q_{1}=q_{2}$ or in other words

$u_{1}=u_{2}+p_{2}-p_{1}$.

Expected profits are $\bar{\Pi}_{\mathrm{H}}=\frac{1}{2 \hat{W}}\left(p_{1}\left(\hat{W}-p_{1}\right)\left(1+u_{1}-\right.\right.$ $\left.u_{2}\right)+p_{2}\left(\hat{W}-p_{2}\right)\left(1-u_{1}+u_{2}\right)+\min \left\{p_{1}, p_{2}\right\}\left|p_{2}-p_{1}\right|$ $\left.\left(u_{1}+u_{2}-1\right)\right)-\beta\left(p_{1}+u_{1}\right)$ or, using Eq. (15),

$$
\begin{aligned}
\bar{\Pi}_{\mathrm{H}}= & \frac{1}{2 \hat{W}}\left(p_{1}\left(\hat{W}-p_{1}\right)\left(1-p_{1}+p_{2}\right)+p_{2}\left(\hat{W}-p_{2}\right)\left(1+p_{1}-p_{2}\right)\right. \\
& \left.+\max \left\{p_{1}\left(p_{2}-p_{1}\right), p_{2}\left(p_{1}-p_{2}\right)\right\}\left(2 u_{2}-1-p_{1}+p_{2}\right)\right) \\
& -\beta\left(p_{2}+u_{2}\right),
\end{aligned}
$$

subject to $p_{2} \in\left[p_{1}-1, p_{1}+1\right] \cap[0, \hat{W}]$ and $u_{2} \in\left[\left(1+p_{1}-\right.\right.$ $\left.\left.p_{2}\right) / 2,1\right]$. Thus, depending on the sign of $\partial_{u_{2}} \bar{\Pi}_{\mathrm{H}}=$ $\frac{1}{\hat{W}} \max \left\{p_{1}\left(p_{2}-p_{1}\right), p_{2}\left(p_{1}-p_{2}\right)\right\}-\beta$, either $u_{2}=1$ (for $\partial_{u_{1}} \bar{\Pi}_{\mathrm{H}}>0$ ) or $u_{2}=\left(1+p_{1}-p_{2}\right) / 2$ (for $\left.\partial_{u_{1}} \bar{\Pi}_{\mathrm{H}}<0\right)$. In the latter case there is no overlap between the two products, which we term "separation" ( $c f$. Fig. 3). In the case where $u_{2}=1$, there clearly is "overlap," and relation (15) entails that then $p_{1} \geq p_{2}$. It is clear that overlap in practice means horizontal "product cannibalization," for some consumers are offered an individually rational choice (satisfying Eqs. (1)-(2)) between both products. It turns out that under ex-ante versioning horizontal overlap is never optimal, as the firm can do better by either eliminating cannibalization or purely vertically differentiating. This finding does not hold under delayed differentiation, where horizontal cannibalization may be optimal as the best possible "compromise" for certain intermediate demand realizations.

Proposition 3. Let the parameters $0<\beta<1$ and $\hat{W}>0$ be given.

(i) Horizontal differentiation with separation yields optimal expected profits of

$\bar{\Pi}_{\mathrm{H}_{1}}^{*}=\frac{\hat{W}}{4}(1-\beta)^{2}-\frac{\beta}{2}=\bar{\Pi}_{1}^{*}+\frac{\beta}{2}$,

with symmetric product portfolio $\left(p_{k}^{*}, q_{k}^{*}\right)=(\hat{W}(1-\beta) /$ $2,(1+\hat{W}(1-\beta)) / 2$, for $k=1,2$.

(ii) There is no horizontal product cannibalization, i.e., optimal expected profits $\bar{\Pi}_{H_{2}}^{*}$ under horizontal differentiation with overlap are dominated,

$\bar{\Pi}_{\mathrm{H}_{2}}^{*}=<\max \left\{\bar{\Pi}_{\mathrm{H}_{1}}^{*}, \bar{\Pi}_{\mathrm{V}}^{*}\right\}=\bar{\Pi}^{*}$.

When considering only horizontal differentiation, Proposition 3 implies that product cannibalization is not inherently ruled out, which may seem counterintuitive in our simple setting. In fact, for lower unit cost of quality $\beta$ overlap (i.e., product cannibalization) performs better than separation, which only yields a constant improvement of $\beta / 2$ over single-product optimal profits $\bar{\Pi}_{1}^{*}$. Cannibalization implies an overinvestment in quality in the sense that more quality is provided than necessary for full horizontal coverage of the consumer base. Exploiting the consumers' feasibility constraint it is possible (for low $\beta$ ) to achieve a vertical separation of the consumer base offering one product at a lower price than the other. The high-price product is then solely preferred by high- $w$ consumers of very good horizontal fit. This feature of the model explains the possibility of price dispersion in a purely horizontally differentiated market when the marginal cost of creating additional quality is sufficiently low. The following result summarizes the firm's entry decision into the market in the absence of an option to delay product differentiation (cf. Fig. 4).

Proposition 4. Consider a firm that has the option to enter the market, given $(\beta, \hat{W}) \in(0,1) \times \mathcal{S}$, with one or two information goods.

(i) For any $(\beta, \hat{W})$ such that

$2 \beta /(1-\beta)^{2}<\hat{W}$,

the firm's entry is viable if versioning costs are small enough. 

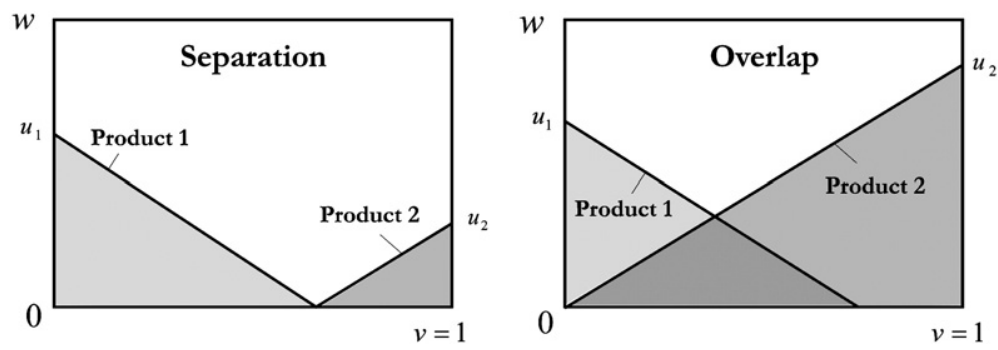

Fig. 3. Horizontal separation and overlap in terms of consumers' net utility.

(ii) In the case that Eq. (19) does not hold, the firm's entry is not viable, even if versioning costs are zero.

(iii) If entry is viable and $\hat{W}<4 \beta /(1-\beta)^{2}$, then the firm needs to enter the market with two products.

An interesting implication from the last proposition is that in some markets for information goods that are unprofitable for any single product, a firm may still be able to enter profitably with a versioned portfolio of products.

\subsection{Effect of versioning costs}

We have seen above that under perfect information mixed differentiation is strictly dominated by pure vertical differentiation. Thus, we can limit ourselves to adjusting and comparing expressions (6), (10), and (29). As a consequence of the earlier discussion, write $\bar{\Pi}_{\mathrm{H}}^{*}$ (instead of $\bar{\Pi}_{\mathrm{H}_{1}}{ }^{*}$ ) for the optimal profits under pure horizontal differentiation (separation). Following Eq. (29), pure horizontal differentiation is dominated by single-product monopoly if and only if $\alpha_{\mathrm{H}}>\beta / 2$. On the other hand, it is dominated by pure vertical differentiation if and only if

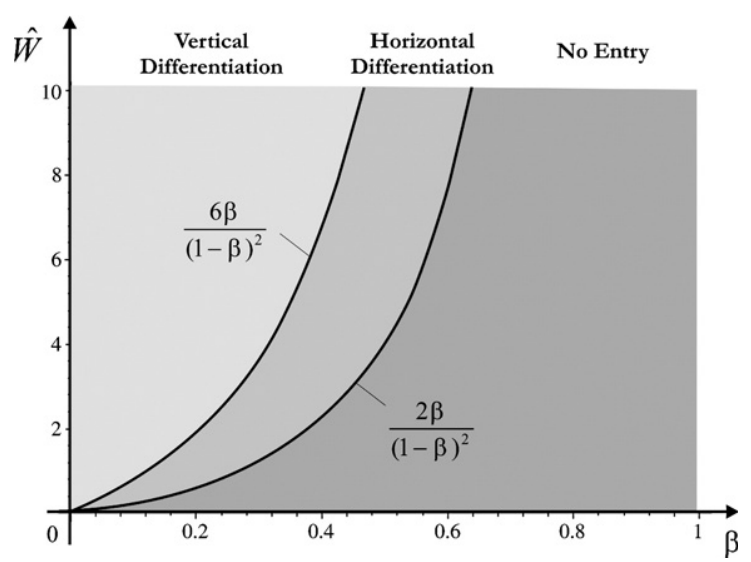

Fig. 4. Optimal ex-ante product versioning.
$\alpha_{\mathrm{V}}<\alpha_{\mathrm{H}}+\frac{\hat{W}}{12}(1-\beta)^{2}-\frac{\beta}{2}$. The resulting partition of the $\left(\alpha_{\mathrm{H}}, \alpha_{\mathrm{V}}\right)$-plane is depicted in Fig. 5.

\section{Delayed differentiation}

Assume that the risk-neutral firm can delay the versioning decision until after the demand uncertainty has resolved through introduction of the flagship product. The exact timing proceeds over two stages $(t \in\{1$, $2\})$ : at $t=1$ the firm develops its flagship product of fixed $^{14}$ attributes $\left(q_{1}, z_{1}\right)$ and presents it to the market without collecting revenues. In the second stage $(t=2)$ firm learns the true value for the maximum reservation price $W$, decides about introducing a versioned product of nonprice attributes $\left(q_{2}, z_{2}\right)$, and prices both products at $p_{1}, p_{2}$ respectively. As a result, the firm needs to choose its product portfolio such that its ex-ante expected profits achieve

$\bar{\Pi}^{* *}=\max _{q_{1}}\left\{E\left[\max \left\{\Pi_{\mathrm{V}}^{*}\left(q_{1} ; W\right), \Pi_{\mathrm{H}}^{*}\left(q_{1} ; W\right)\right\}\right]\right\}$

instead of the $\bar{\Pi}^{*}=\max \left\{\bar{\Pi}_{\mathrm{V}}^{*}, \bar{\Pi}_{\mathrm{H}}^{*}\right\}$ it obtains under exante versioning according to Proposition 3 . We solve this problem in stages, beginning with the second stage.

\subsection{Optimal ex-post versioning}

Assume the firm has observed the realization of $W$ and has decided about the quality $q_{1}$ of its flagship product. As in Section 3, it is possible to differentiate the versioned product either vertically $\left(z_{2}=0\right)$ or horizontally $\left(z_{2}=1\right)$, provided that its quality does not exceed the

\footnotetext{
${ }^{14}$ Quality upgrades are not possible for $t>1$, since e.g., the development team has been disbanded or upfront costs to restart significant quality improvements are prohibitive. The firm never has an incentive to change the horizontal nonprice attribute of the flagship product, $z_{1}=0$.
} 


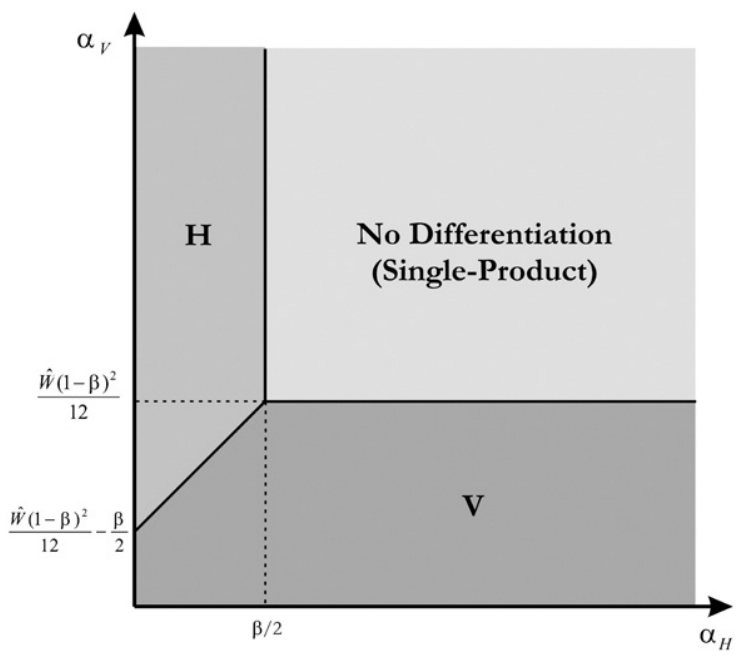

Fig. 5. Optimal modes of ex-ante differentiation for versioning cost tuples $\left(\alpha_{\mathrm{H}}, \alpha_{\mathrm{V}}\right) \geq 0$.

flagship product's, i.e., $q_{1} \geq q_{2}$ ( $c f$. footnote 14). Alternately the firm has the option not to introduce a vertically versioned product at all. ${ }^{15}$

\subsubsection{Pure vertical differentiation}

Consider the case when $z_{2}=0$ and the resulting portfolio contains two products. Without loss of generality we can assume that $u_{1}^{*}=u_{2}^{*}=q_{1}-p_{1}^{*}$, since - as long as they are free - larger values for $u$ weakly increase profits. The profit-maximization problem becomes

$$
\begin{aligned}
\max _{p_{1}, p_{2}} & \prod_{\mathrm{V}}\left(p_{1}, p_{2} ; q_{1}, W\right) \\
& =\max _{p_{1}, p_{2}}\left\{\frac{p_{1}\left(W-p_{1}\right)+p_{2}\left(p_{1}-p_{2}\right)}{W} \min \left\{1, q_{1}-p_{1}\right\}-\beta q_{1}\right\}
\end{aligned}
$$

with solution equivalent to that of the ex-ante versioning problem, as long as $q_{1}$ is large enough so that full horizontal coverage holds. If on the other hand $q_{1}$ is small compared to the realization $W$, then horizontal coverage will not be full and product breadth is traded for the ability to charge high prices. In other words, "transportation costs" for consumers with significant misfit will be too

\footnotetext{
${ }^{15}$ The firm could also reprice the first product out of the market (at $p_{1} \geq W$ ) and vertically version a second product, which is, however, strictly inferior when the versioning $\operatorname{cost} \alpha_{\mathrm{V}}$ is positive. If the firm does not introduce the second product at all, then it needs to solve $\max _{p_{1}^{\prime} \in[0, W]}$ $p_{1}^{\prime}\left(W-p_{1}^{\prime}\right) \min \left\{1, q_{1}-p_{1}^{\prime}\right\}$, with solution $p_{1}^{\prime *}$ that can be obtained by replacing $W$ with $3 W / 4$ in Eq. (21). The firm does not incur a versioning cost, so that at least for large $\alpha_{\mathrm{V}}$ not introducing a second product is optimal, given that vertical differentiation is considered. If on the other hand $\alpha_{V}=0$ (or $\alpha_{V}>0$, but small enough), then introducing the versioned product in the second round is a good idea, since it can always be accomplished by setting $p_{2}=0$.
}

high to buy any of the products. More specifically we obtain $\left(p_{2}^{*}, q_{2}^{*}\right)=\left(p_{1}^{*} / 2, q_{1}-p_{1}^{*} / 2\right)$, and

$p_{1}^{*}= \begin{cases}2 W / 3, & \text { if } q_{1} \geq 1+2 W / 3, \\ q_{1}-1, & \text { if } q_{1} \in\left[\bar{q}_{\mathrm{V}}(W), 1+2 W / 3\right], \\ \left(4 W+3 q_{1}-\sqrt{\left(4 W-3 q_{1}\right)^{2}+12 W q_{1}}\right) / 9, & \text { otherwise. }\end{cases}$

where $\bar{q}_{\mathrm{V}}(W)=\left(6+2 W-\sqrt{9+4 W^{2}}\right) / 3 \in[1, \min \{2,1+$ $2 W / 3\}]$. From this one can derive an expression for the optimal profits as a function of $q_{1}$,

$\Pi_{\mathrm{V}}^{*}\left(q_{1}, W\right)= \begin{cases}W / 3-\beta q_{1}, & \text { if } q_{1} \geq 1+2 W / 3, \\ \left(q_{1}-1\right)\left(1-\frac{3\left(q_{1}-1\right)}{4 W}\right)-\beta q_{1}, & \text { if } q_{1} \in\left[\bar{q}_{\mathrm{V}}(W), 1+2 W / 3\right], \\ p_{1}^{*}\left(1-\frac{3 p_{1}^{*}}{4 W}\right)\left(q_{1}-p_{1}^{*}\right)-\beta q_{1} . & \text { otherwise. }\end{cases}$

Due to the cost complementarities a versioned product will always be introduced as long as the versioning cost $\alpha_{\mathrm{V}}$ is small enough. The development cost for the flagship product is already sunk and a second product can then only increase profits (at least as long as $\alpha_{\mathrm{V}}, \alpha_{\mathrm{H}}$ are small enough). It is straightforward to show that for any $W>0$ the profit function $\Pi_{\mathrm{V}}^{*}\left(q_{1} ; W\right)$ is continuously differentiable in $q_{1}>0$ with $\partial_{q 1} \Pi_{\mathrm{V}}^{*}(0)=\partial_{q 1} \Pi_{\mathrm{V}}^{*}(1+2 W /$ $3))=-\beta<\partial_{q 1} \Pi_{\mathrm{V}}^{*}\left(\overline{\mathrm{q}}_{\mathrm{V}}\right)=\left(\sqrt{4 W^{2}+9}-3\right) /(2 W)-\beta$. In addition, the state-contingent profit function $\Pi_{\mathrm{V}}^{*}$ has at most one maximum in $q_{1}>0$ but is generally not quasiconcave. $^{16}$

\subsubsection{Horizontal differentiation (including mixed)}

When deciding to horizontally differentiate, the firm chooses $z_{2}=1$ and then needs to decide about $p_{1}, p_{2}$ and $q_{2}=u_{2}+p_{2} \leq u_{1}+p_{1}=q_{1}$. As in the ex-ante versioning problem ( $c f$. Section 3) there are two general cases, "separation" and "overlap." The details of the overlap case, which is synonymous with "horizontal product cannibalization," are discussed in the Appendix, where analytical solutions are provided. Under delayed differentiation it is indeed sometimes best to horizontally cannibalize products, whereas this is never optimal under ex-ante versioning (Proposition 5). Independent of the versioning costs, it is never optimal to version along both horizontal and vertical attributes at the same time. ${ }^{17}$

There are four possible configurations that all exhibit horizontal separation, depending on how large the

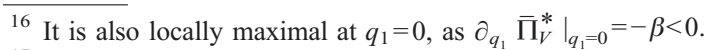

17 This finding is somewhat driven by the quasi-linearity of the model in preferences and the assumption that the consumers are uniformly distributed. Including nonlinearities in the consumers' preferences, interior solutions favoring simultaneous differentiation with respect to more than one attribute cannot be ruled out.
} 
quality $q_{1}$ is with respect to the demand realization $W$ : (i) if $q_{1} \geq 1+W / 2$ (i.e., $q_{1}-p_{1} \geq 1$ ), given separation there is just one product and $p_{1}^{*}=W / 2$ analogous to the singleproduct firm. (ii) If $1 / 2 \leq q_{1}-p_{1}=1-\left(q_{2}-p_{2}\right)<1$, then

$$
\begin{aligned}
\left(p_{1}^{*}, p_{2}^{*}\right)= & \arg \max _{p_{1}, p_{2}}\left\{\frac{p_{1}\left(W-p_{1}\right)\left(q_{1}-p_{1}\right)}{2 W}\right. \\
& \left.+\frac{p_{2}\left(W-p_{2}\right)\left(1-q_{1}+p_{1}\right)}{2 W}-\beta q_{1}\right\}=\left(\frac{W}{2}, \frac{W}{2}\right),
\end{aligned}
$$

for $q_{1} \in[(1+W) / 2,1+W / 2]$. Note that in this case $q_{2}^{*}=1+\mathrm{W}-q_{1}$, resulting in mixed differentiation without price dispersion. (iii) If $q_{1} \in\left[\bar{q}_{\mathrm{H}}(W),(1+W) / 2\right]$, then $p_{1}^{*}=p_{2}^{*}=q_{1}-1 / 2$, and $q_{2}^{*}=q_{1}$ (pure horizontal differentiation). Thereby $\bar{q}_{\mathrm{H}}(W)=\left(2+W-\sqrt{1+W^{2}}\right) / 2 \in$ $[1 / 2, \min \{1,(1+W) / 2\}]$. (iv) If $q_{1}-p_{1}<1-\left(q_{2}-p_{2}\right)$ (equivalent to $q_{1}-p_{1}<1 / 2$ ), then $p_{1}=p_{2}$ and $q_{1}=q_{2}$, since the situation is symmetric (pure horizontal differentiation). We have

$$
\begin{aligned}
p_{1}^{*} & =\arg \max _{p_{1}}\left\{\frac{p_{1}\left(W-p_{1}\right)\left(q_{1}-p_{1}\right)}{W}-\beta q_{1}\right\} \\
& =\left(W+q_{1}-\sqrt{\left(W-q_{1}\right)^{2}+W q_{1}}\right) / 3
\end{aligned}
$$

for $q_{1}<\bar{q}_{\mathrm{H}}(W)$. Summarizing these results we obtain

$$
\Pi_{\mathrm{H}_{1}}^{*}\left(q_{1} ; W\right)= \begin{cases}W / 4-\beta q_{1}, & \text { if } q_{1} \geq(1+W) / 2, \\ \left(q_{1}-1 / 2\right)\left(1-\left(q_{1}-1 / 2\right) / W\right)-\beta q_{1}, & \text { if } q_{1} \in\left[\overline{\mathrm{q}}_{\mathrm{H}},(1+W) / 2\right], \\ 2 p_{1}^{*}\left(1-p_{1}^{*} / W\right)\left(q_{1}-p_{1}^{*}\right)-\beta q_{1}, & \text { otherwise. }\end{cases}
$$

In the case of realizations $W$ such that $q_{1} \in[(1+W) / 2$, $1+W / 2]$ the firm would use mixed versioning; however, the following proposition shows that this is never optimal. Analogous to the pure-vertical-versioning case, it can be shown that the profit function $\Pi_{\mathrm{H}_{1}}$ is continuously differentiable and has at most one maximum in $q_{1}>0$.

Proposition 5. (i) For any given realization of $W$ in $\mathcal{S}$, either pure horizontal or pure vertical differentiation is optimal. (ii) Horizontal product cannibalization (overlap) may be optimal for certain "intermediate" realizations of $W$.

Horizontal overlap in the product portfolio is most likely to be useful if the support $\mathcal{S}$ of demand realizations is such that for low states $W$ vertical differentiation is optimal while for high states horizontal separation is best. Note that in the presence of horizontal product cannibalization, there generally is price dispersion ( $c f$. the details on the overlap case in the Appendix). Figs. 6 and 7 show the optimal firm profits and modes of ex-post differentiation for $\mathcal{S}=[1,2]$ and $\beta=1 / 10$.

\subsubsection{Effect of versioning costs}

As a consequence of Proposition 5 mixed versioning cost, $\alpha_{M} \geq \alpha_{H}, \alpha_{V}$, will never be incurred. With perfect ex-ante information about $W$, the effect of versioning cost is of course adequately displayed in Fig. 5 with $\hat{W}$ replaced by $W$. In the presence of uncertainty, the optimal policy depends on the magnitudes and the difference between $\alpha_{\mathrm{V}}$ and $\alpha_{\mathrm{H}}$ as these influence the attractiveness of one versioning mode over the other and of product-line extensions overall.

\subsection{The product development decision}

In the first stage of the versioning problem with delayed differentiation the firm decides about the appropriate development effort (i.e., investment in product quality $q_{1}^{* *}$ ) by solving problem (20). As pointed out in Section 1, irreversibilities in investments lead firms to value the option of delaying commitment $[22,28,38]$. Under delayed differentiation in the presence of demand uncertainty the marginal value of quality may be higher than under ex-ante versioning where an adaptation of the product portfolio to the realization of the uncertain demand is not possible.

Proposition 6. If the optimal ex-post versioning policy is not state-contingent, i.e., the mode of differentiation does not depend on $W$, then the optimal ex-ante investment $q_{1}^{* *}$ is characterized by ${ }^{18}$

$$
q_{1}^{* *}=1+\frac{2}{3} \frac{\int_{W_{1}\left(q_{1}^{* *}\right)}^{W_{2}\left(q_{1}^{* *}\right)} f(w) \mathrm{d} w-\beta+\int_{W_{2}\left(q_{1}^{* * *}\right)}^{\bar{s}} p_{1}^{*}(w)\left(1-\frac{3 p_{1}^{*}(w)}{4 w}\right) f(w) \mathrm{d} w}{\int_{W_{1}\left(q_{1}^{* *}\right)}^{\left.W_{2}^{* *}\right)} \frac{(w)}{w} \mathrm{~d} w}
$$

for pure vertical differentiation (with $\bar{s}=\max \{\mathcal{S}\}, W_{1}=3$ $\left(q_{1}^{* *}-1\right) / 2, \bar{q}_{V}\left(W_{2}\right)=q_{1}^{* *}$, and $p_{1}^{*}$ as in Eq. (21) for $w=W)$, and

$q_{1}^{* *}=\frac{1}{2}+\frac{\int_{W_{1}\left(q_{1}^{* *}\right)}^{W_{2}\left(q_{1}^{* *}\right)} f(w) \mathrm{d} w-\beta+2 \int_{W_{2}\left(q_{1}^{* *}\right)}^{s} p_{1}^{*}(w)\left(1-\frac{p_{1}^{*}(w)}{w}\right) f(w) \mathrm{d} w}{2 \int_{W_{1}\left(q_{1}^{* *}\right)}^{W_{2}\left(q_{1}^{* *}\right)} \frac{f(w)}{w} \mathrm{~d} w}$

for horizontal differentiation without overlap (with $\bar{s}$ as above, $W_{1}=2 q_{1}^{* *}-1, \bar{q}_{H_{1}^{*}}^{* *}\left(W_{2}\right)=q_{1}^{* *}$, and $p_{1}^{*}$ as in Eq. (23) for $w=W$ ).

\footnotetext{
${ }^{18}$ It is clear that horizontal differentiation with overlap can never be the result of a non-state-contingent versioning policy.
} 


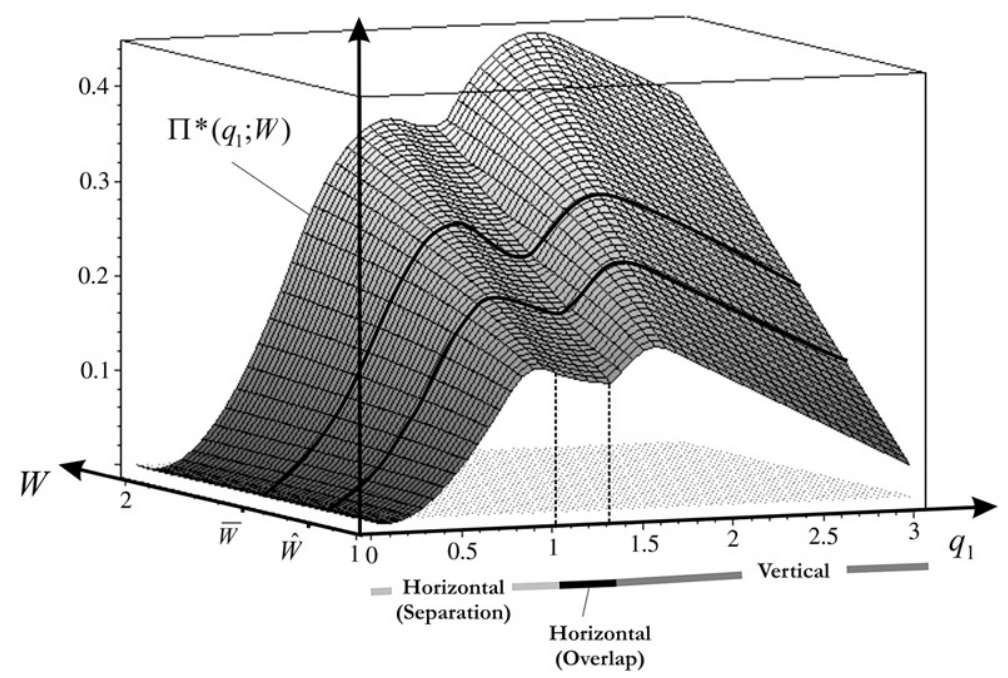

Fig. 6. Optimal cumulative profits as a function of $q_{1}$ and $W$ (for $\beta=1 / 10, \mathcal{S}=[1,2]$ ).

If the uncertainty about the demand characteristic $W$ is low, then $\left[W_{1}, W_{2}\right] \cap \mathcal{S}=\mathcal{S}$, so that the expressions (25) and (26) are identical to the optimal ex-ante quality choice $q_{1}^{*}$ under vertical and horizontal differentiation respectively ( $c f$. Section 3.2). If the development $\operatorname{cost} \beta$ is zero, then clearly it is optimal to vertically version and choose $q_{1}^{* *}=1+2 \bar{s} / 3$, a quality larger than the ex-ante optimal quality of $q_{1}^{*}=1+2 \hat{W} / 3$ for $\beta=0$, since uncertainty about demand implies uncertainty about the optimal pricing of the product portfolio. Hence, even if quality can be provided at an arbitrarily small positive cost, a firm lacking the flexibility to version and to reprice according to observed demand will generally limit its flagship product's quality.

\subsubsection{Example}

For state-contingent versioning policies a firm's investment in product development does not necessarily increase. Consider the case when the support $\mathcal{S}$ is finite containing only the positive elements $W_{\mathrm{L}}=2$ and $W_{\mathrm{H}}=4$, which correspond to a "low" and a "high" demand realization respectively. The probability of high demand is denoted by $\pi_{\mathrm{H}}$ and the development cost is $\beta=1 / 4$. Thus, the ex-ante demand estimator is $\hat{W}=4 /\left(2-\pi_{\mathrm{H}}\right)$, which satisfies the viability condition (19) for all $\pi_{\mathrm{H}} \in[0,1]$. For $\hat{W}$ less than $6 \beta /(1-\beta)^{2}=8 / 3$ (i.e., $\left.\pi_{\mathrm{H}}<1 / 2\right)$ the firm decides ex-ante to horizontally version, whereas for $\pi_{\mathrm{H}}>1 / 2$ vertical versioning is optimal. Under delayed differentiation the firm prefers to not commit to any particular mode of differentiation for a large range of intermediate $\pi_{\mathrm{H}}$ 's. In the case of a "surprisingly" high realization of demand the ex-ante quality choice is relatively too small, so that horizontal versioning is optimal. The converse is true for a low demand realization, when it is better to vertically version in order to make use of the "unexpectedly" high flagship quality. Fig. 8 shows that the firm's development effort (proportional to $q_{1}$ ) may go both up and down with delayed differentiation as a result of actively using its added flexibility in the form of a state-contingent horizontal-vertical versioning policy.

\subsubsection{Value of perfect information}

The expected value of perfect information (EVPI) about the demand under ex-ante versioning is simply the difference between ex-ante optimal profit evaluated at $W$

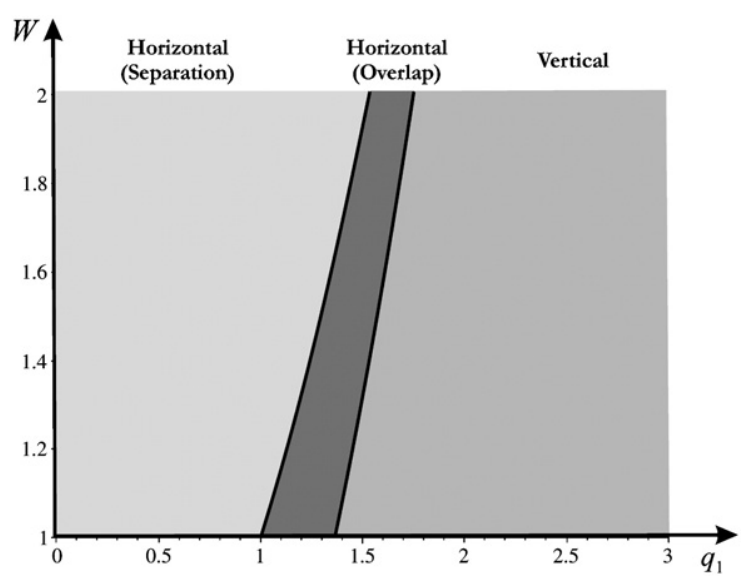

Fig. 7. Optimal modes of ex-post differentiation (for $\beta=1 / 10, \mathcal{S}=[1,2]$ ). 


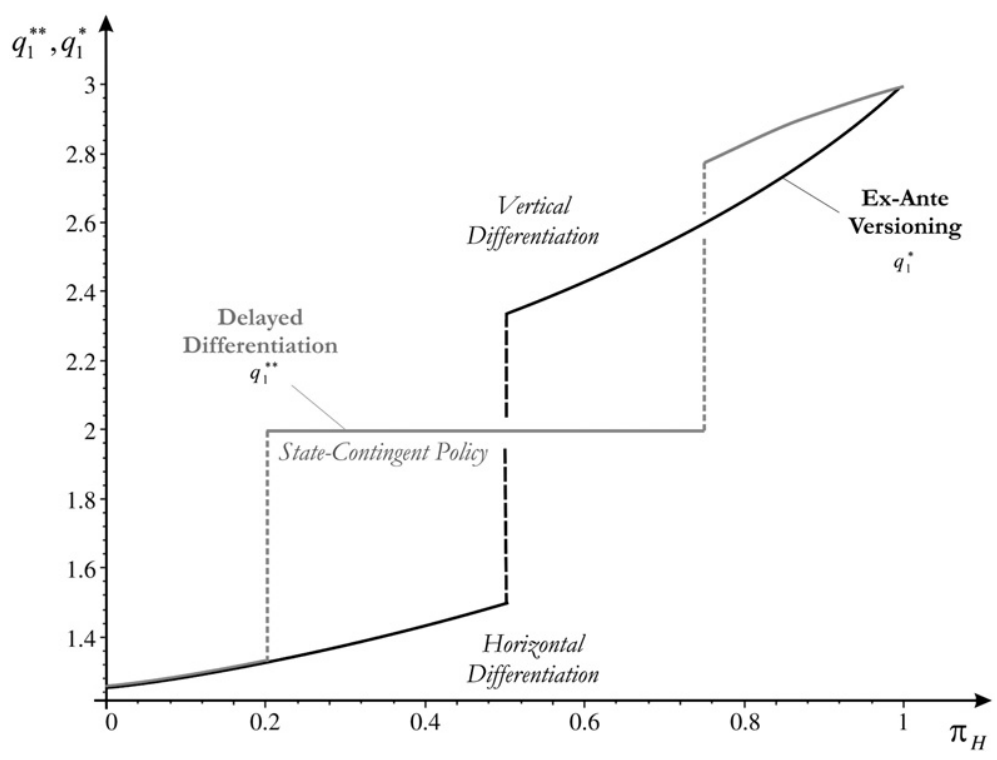

Fig. 8. Optimal choice of $q_{1}$ under ex-ante versioning and under delayed differentiation (for $\beta=1 / 4, \mathcal{S}=\{2,4\}$ ).

(in expectation) and evaluated at $\hat{W}$ respectively: EVPI (ex-ante versioning $)=E \Pi^{*}(W)-\Pi^{*}(\hat{W})$, where

$\Pi^{*}(W)= \begin{cases}W(1-\beta)^{2} / 3-\beta, & \text { if } W \geq 6 \beta /(1-\beta)^{2}, \\ W(1-\beta)^{2} / 4-\beta / 2, & \text { if } W \in\left[2 \beta /(1-\beta)^{2}, 6 \beta /(1-\beta)^{2}\right] \\ 0, & \text { otherwise. }\end{cases}$

Clearly EVPI is an upper bound for the value of the option to delay differentiation [9]. It is generally not monotonic in the development $\operatorname{cost} \beta$, as the way in which information alters the versioning decision depends on the firm's default no-information versioning policy.

\subsubsection{Value of the option to delay differentiation}

The option value of not having to commit to the product portfolio ex ante is given by $V_{\mathrm{d}}=\bar{\Pi}^{* *}-\bar{\Pi}^{*}$. This value must be zero for large values of $\beta$, since both $\bar{\Pi}^{* *}$ and $\bar{\Pi}^{*}$ are tightly bounded from below by zero and are strictly decreasing (and continuous) in $\beta$.

Proposition 7. (i) The value of the option to delay differentiation, $V_{d}(\beta)$, is locally maximal at $\beta \in\{0,(3+$ $\hat{W}-\sqrt{9+6 \hat{W}}) / \hat{W}\}$. (ii) Its slope with respect to the development cost $\beta$ is given almost everywhere by the difference of the optimal flagship qualities with and without delayed differentiation,

$\partial_{\beta} V_{\mathrm{d}} \stackrel{\text { a.e. }}{=} q_{1}^{*}-q_{1}^{* *}$.

(iii) The option value is zero for large $\beta$ 's.
Development costs in which the firm is ex ante indifferent between vertical and horizontal versioning make the option to delay decisions about the composition of the product portfolio particularly valuable. A state-contingent policy specifying the mode of differentiation as a function of the realized demand clearly outperforms the noncontingent ex-ante decision. In particular, the value of flexibility is generally non-

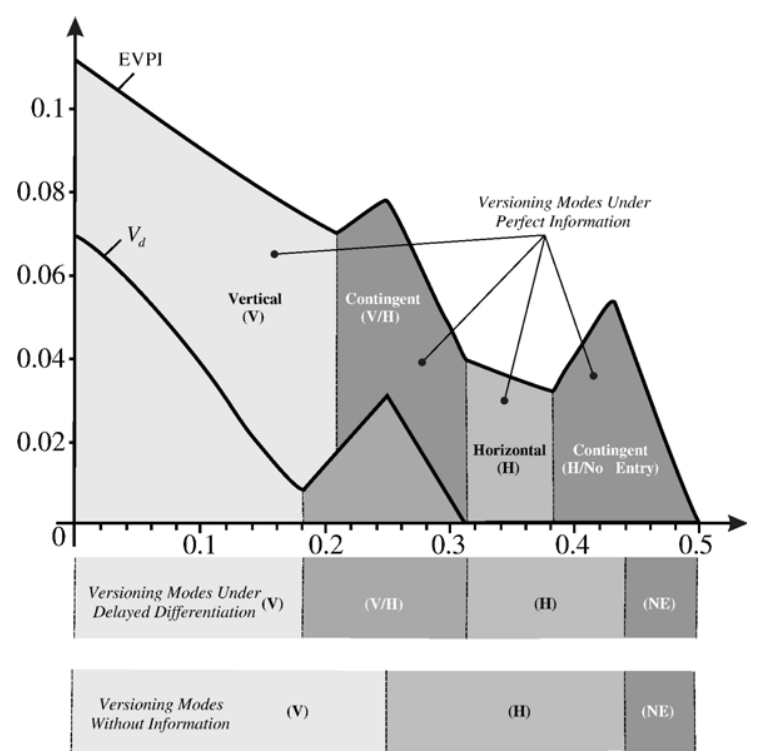

Fig. 9. Expected value of perfect information (EVPI) and value of the option to delay differentiation $\left(V_{\mathrm{d}}\right)$ as a function of the development $\operatorname{cost} \beta$ (for $\mathcal{S}=\{2,4\}, \pi_{\mathrm{H}}=.5$ ). 
monotonic in quality cost; it depends on the way in which a deviation from the firm's default ex-ante strategy influences expected profits. Varying the development cost, the option value varies proportional to the difference $q_{1}^{*}-q_{1}^{* *}$, which can be positive and negative. Fig. 9 depicts the option value for our previous two-state example (with $\mathcal{S}=\left\{W_{\mathrm{L}}, W_{\mathrm{H}}\right\}$ ) together with the expected value of perfect information as a function of $\beta$.

\subsection{Effects on consumer surplus}

Consumer surplus depends directly on the firm's product portfolio and can be directly determined from the set $\Omega=\left\{\left(p_{k}, q_{k}, z_{k}\right)\right\}_{k=1,2}$.

Proposition 8. (i) Given a demand realization $W$ and the firm's product portfolio $\Omega$, the consumer surplus is given in Table 1 (ii) Under ex-ante optimal versioning, the expected consumer surplus is given by

$\mathrm{CS}= \begin{cases}(2+\beta) / 6, & \text { if } \beta \leq \beta^{\prime}, \\ (1+\beta) / 8, & \text { if } \hat{W} \in\left[\beta^{\prime}, \beta^{\prime \prime}\right], \\ 0, & \text { otherwise. }\end{cases}$

where $\beta^{\prime}=(3+\hat{W}-\sqrt{9+6 \hat{W}}) / \hat{W}$ and $\beta^{\prime \prime}=(1+\hat{W}-$ $\sqrt{1+2 \hat{W}}) / \hat{W}$.

The first part of Proposition 8 describes how consumer surplus can be determined from the results in Sections 4.1 and 4.2. The second part shows that under ex-ante differentiation consumer surplus is piecewise linearly increasing in $\beta$. At the points of discontinuity $\beta^{\prime}$ and $\beta^{\prime \prime}$, where the first mode of differentiation changes and then the firm decides not to enter the market, consumer surplus drastically decreases. For each given versioning mode, consumer surplus is increasing in $\beta$

Table 1

Expected consumer surplus given a two-element product portfolio $\Omega$

\begin{tabular}{ll}
\hline Mode of differentiation & Consumer surplus $\left[u_{k}=q_{k}-p_{k}, \delta=p_{1}-p_{2}\right]$ \\
\hline Vertical & $\frac{W-p_{2}}{W}\left(u_{1}-\min \left\{u_{1}, 1\right\} / 2\right) \min \left\{u_{1}, 1\right\}$ \\
$\begin{array}{l}\text { Horizontal } \\
\text { (separation) }\end{array}$ & $\frac{W-p_{1}}{W}\left(u_{1}-\min \left\{u_{1}, \frac{1}{2}\right\} / 2\right) \min \left\{u_{1}, \frac{1}{2}\right\}$ \\
$\begin{array}{c}\text { Horizontal } \\
\text { (overlap) }\end{array}$ & $\frac{W-p_{1}}{W}\left(u_{1}-\frac{1-\delta}{4}\right) \frac{1-\delta}{2}$ \\
& $+\frac{W-p_{2}}{W}\left(u_{2}-\frac{1+\delta}{4}\right) \frac{1+\delta}{2}$ \\
& $+\frac{\delta}{W}\left(u_{2}-\frac{\min \left\{u_{2}, 1\right\}}{2}\right) \min \left\{u_{2}, 1\right\}$
\end{tabular}

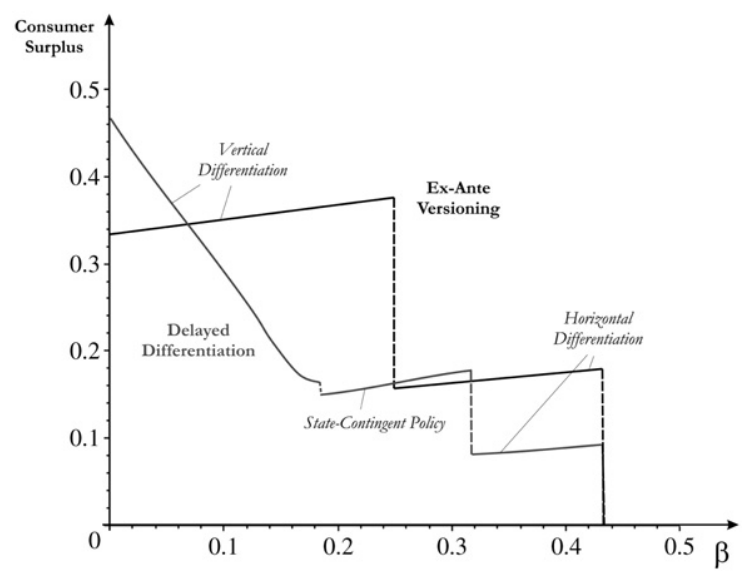

Fig. 10. Consumer surplus (CS) as a function of the development cost $\beta$ (for $\left.\mathcal{S}=\{2,4\}, \pi_{\mathrm{H}}=.5\right)$.

because the firm compensates for its decreasing quality investment by reducing price so as to ensure a large participation. In fact, expected market penetration ${ }^{19}$ increases between two and four times faster than consumer surplus.

As shown above, the firm generally values versioning flexibility, which may both increase or decrease the optimal ex-ante quality investment depending on the noinformation default strategy. Interestingly, the increased profitability of the firm's investment does not always imply a decrease in consumer surplus. For low development cost, the benefits of the higher flagship quality may outweigh the firm's improved ability to screen the consumer base, leading for low $\beta$ 's to a higher consumer surplus under delayed differentiation than under ex-ante versioning (cf. Fig. 10).

\subsection{Generalization to goods with positive marginal costs}

A generalization of virtually all results in this paper to goods with positive marginal costs $c>0$ can be achieved via a simple variable transformation. The only restrictive assumption is that the marginal costs for both goods be the same. This is realistic for many products, and especially those where the actual versioning decision can be taken far downstream in the supply chain [1], e.g., by the retailer. Replacing the prices $p_{k}$ in Section 3 by the markups $m_{k}=p_{k}-c$ (for $k=1,2$ ), together with using the constants $\hat{W}_{c}=\hat{W}-c$ and $\beta_{c}=\beta \hat{W} /(\hat{W}-c)$ is enough for

\footnotetext{
${ }^{19}$ Expected market penetration under ex-ante versioning is $(1+\beta) /$ $2 \in[1 / 2,1]$ for a horizontally and $(2+\beta) / 3 \in[2 / 3,1]$ for a vertically differentiated optimal product portfolio.
} 
all profit-maximization problems under ex-ante versioning to carry over to the case with $c>0$. Under delayed differentiation the same change of variables can be used; the estimator $\hat{W}$ needs only to be replaced by the actual demand realization $W$. In addition, the firm needs to consider $q_{1}^{\prime}=q_{1}-c$ for its second-stage versioning decision. Again all of the maximizers can be simply recovered by substituting the new constants. The overall effect of these transformations is that positive marginal costs are equivalent to increasing the development costs and also add an additional fixed cost of entry, changing the firm's viability requirements for market entry.

\section{Conclusion and further research}

Even when a company can extend its product line by varying horizontal and vertical attributes of a flagship product, it is - given our assumptions of quasilinear preferences and homogeneous consumer distribution not optimal to do both at the same time. ${ }^{20}$ This finding is independent of the versioning cost structure, as long as $\alpha_{M} \geq \alpha_{V}, \alpha_{H}$. Nevertheless, under delayed differentiation the firm's optimal versioning policy contingent on the demand realization generally incorporates both modes of differentiation. For low demand realizations it is best to differentiate vertically, adding a product of degraded quality to the then relatively high-performance flagship product to more adequately segment the consumer base by maintaining full horizontal market coverage. This can be accomplished by deliberately "damaging" the flagship product [11] to increase the product portfolio. On the other hand, for (unexpectedly) large demand realizations horizontal differentiation is generally superior, since the effective flagship product quality may not be high enough to guarantee full horizontal market coverage. For intermediate realizations of demand, horizontal product cannibalization may be the best compromise. The latter generally implies price dispersion for products of equal quality. Despite the quite general insight from option theory that added flexibility usually leads to an increase of a firm's ex-ante investment which has become "less irreversible," we find that for multiattribute versioning the upfront product development efforts may decrease if, due to the possibility of delayed differentiation, a policy contingent on the demand state becomes optimal. This result is driven by the nonconvexity of the problem,

\footnotetext{
${ }^{20}$ This finding cannot be expected to hold for product portfolios with more than two products, the treatment of which in our framework poses significant analytical difficulties. It is also not necessarily what is observed in practice ( $c f$. footnote 1 ).
}

horizontal and vertical differentiation yielding locally optimal profits. $^{21}$

Examining the option value of delayed differentiation further, we show that it contains a local maximum at the point of ex-ante indifference between the versioning modes, and that it naturally vanishes for very high product development costs. The effect of delayed differentiation on consumer surplus is mixed. Under ex-ante versioning consumer surplus heavily depends on the mode of differentiation used, and for each mode is monotonically increasing in the firm's development cost, as the firm tends to compensate an increase in quality cost $\beta$ by lowering price and enlarging the consumer base. If differentiation is delayed, the versioning modes switch at different points, so that effects on consumer surplus are ambiguous. Overall it tends to decrease as the firm's ability to make effective pricing and product-lineextension decisions greatly increases.

Future research could proceed along the following three promising axes: (1) extend the approach to more than two products. One of the main challenges here is that the potentially multiple overlaps are hard to capture analytically; (2) admit more general cost functions and demand distributions; and (3) incorporate competitive dynamics into the multiattribute versioning decision.

\section{Appendix A}

Proof of Lemma 1. (i) This part is trivial. (ii) The probability density of $W$ is by assumption given by the Lebesgue-measurable function $f \in L_{1}(\mathcal{S})$. Thus, we have using Fubini's theorem:

$$
\begin{aligned}
\frac{\bar{W}}{\hat{W}} & \left.=\int_{\mathcal{S}} x f(x) \mathrm{d} x\right)\left(\int_{\mathcal{S}} \frac{f(y)}{y} \mathrm{~d} y\right) \\
& =\int_{\mathcal{S} \times \mathcal{S}} \frac{x}{y} f(x) f(y) \mathrm{d}(x, y) \\
& =\int_{\mathcal{S} \times \mathcal{S}} \frac{1}{2}\left(\frac{x}{y}+\frac{y}{x}\right) f(x) f(y) \mathrm{d}(x, y) \\
& >\int_{\mathcal{S} \times \mathcal{S}} f(x) f(y) \mathrm{d}(x, y)=1,
\end{aligned}
$$

since $(x / y+y / x) / 2>1$ for all $x, y>0$ with $x \neq y$. Note that the set of points $(x, y) \gg 0$ where $x=y$ is of measure zero in $\mathcal{S} \times \mathcal{S}$, so that the strict inequality is warranted.

\footnotetext{
${ }^{21}$ From a technical point of view, the maximizer (i.e., the "optimal policy") is upper semicontinuous, essentially as a consequence of the continuity of the objective function. This is guaranteed by Berge's maximum theorem [5].
} 
Proof of Proposition 3. (i) Since there is no overlap, the expected profits under horizontal differentiation (16) are under separation

$$
\begin{aligned}
\bar{\Pi}_{\mathrm{H}_{1}}\left(p_{1}, p_{2}\right)= & \frac{1}{2 \hat{W}}\left(p_{1}\left(\hat{W}-p_{1}\right)\left(1-p_{1}+p_{2}\right)\right. \\
& \left.+p_{2}\left(\hat{W}-p_{2}\right)\left(1+p_{1}-p_{2}\right)\right) \\
& -\beta\left(1+p_{1}+p_{2}\right) / 2 .
\end{aligned}
$$

Note that $\bar{\Pi}_{\mathrm{H}_{1}}\left(p_{1}, p_{2}\right)=\bar{\Pi}_{\mathrm{H}_{1}}\left(p_{2}, p_{1}\right)$ and therefore, if there is an asymmetric maximizer, there must be at least two of them. Hence, without loss of generality, assume that $p_{1} \geq p_{2}$. Let us now introduce the variable $\delta=p_{1}-$ $p_{2} \in[0,1]$, which leads to the problem of maximizing

$$
\begin{aligned}
\bar{\Pi}_{\mathrm{H}_{1}}\left(p_{2}+\delta, p_{2}\right)=- & \frac{p_{2}^{2}}{\hat{W}}+\left(1-\beta-\frac{\delta(1-\delta)}{\hat{W}}\right) p_{2} \\
& +\frac{1}{2}\left(\delta(1-\delta)\left(1-\frac{\delta}{\hat{W}}\right)-\beta(1+\delta)\right)
\end{aligned}
$$

with respect to $p_{2} \in[0, \hat{W}]$ and $\delta \in[0,1]$, subject to the additional constraint $0 \leq p_{2}+\delta \leq \hat{W}$. Neglecting the latter constraint, the first-order condition with respect to $p_{2}$ is $\partial_{p_{2}} \bar{\Pi}_{\mathrm{H}_{1}}\left(p_{2}+\delta, p_{2}\right)=-\frac{2 p}{\hat{W}}+1-\beta-\frac{\delta(1-\delta)}{\hat{W}}=0$, which yields $p_{2}^{*}(\delta)=\frac{\hat{W}}{2}(1-\beta)-\frac{\delta(1-\delta)}{2} \leq \frac{W}{2}(1-\beta)$. Thus,

$$
\begin{aligned}
\bar{\Pi}_{\mathrm{H}_{1}}\left(p_{2}^{*}(\delta)+\delta, p_{2}^{*}(\delta)\right)= & \frac{\hat{W}}{4}(1-\beta)^{2}-\frac{\beta}{2} \\
& -\frac{\delta^{2}}{4 \hat{W}}\left(1-\delta^{2}+2 \beta \hat{W}\right) \\
& \leq \frac{\hat{W}}{4}(1-\beta)^{2}-\frac{\beta}{2} .
\end{aligned}
$$

The last inequality is binding if and only if $\delta=0$. The unique maximizers of the original maximization in the "separation" case, now satisfying all of the above constraints, are $p_{1}^{*}=p_{2}^{*}=\frac{\hat{W}}{2}(1-\beta)$, yielding optimal profits

$\bar{\Pi}_{\mathrm{H}_{1}}^{*}=\frac{\hat{W}}{4}(1-\beta)^{2}-\frac{\beta}{2}=\bar{\Pi}_{1}^{*}+\frac{\beta}{2}$.

These profits are by the amount $\beta / 2$ larger than profits for the single-product monopoly and are positive (i.e., viable) if and only if

$\hat{W}>\frac{2 \beta}{(1-\beta)^{2}}$.

In particular, a multiproduct monopolist firm can substitute horizontal differentiation for a quality increase, and save on cost (provided that $\alpha_{\mathrm{H}}<\beta / 2$ ). (ii) When there is overlap, the expected profit function (16) becomes

$$
\begin{aligned}
\bar{\Pi}_{\mathrm{H}_{2}}\left(p_{1}, p_{2}\right)= & \frac{1}{2 \hat{W}}\left(p_{1}\left(\hat{W}-p_{1}\right)\left(1-p_{1}+p_{2}\right)\right. \\
& +p_{2}\left(\hat{W}-p_{2}\right)\left(1+p_{1}-p_{2}\right) \\
& +\max \left\{p_{1}\left(p_{2}-p_{1}\right), p_{2}\left(p_{1}-p_{2}\right)\right\} \\
& \left.\times\left(1-p_{1}+p_{2}\right)\right)-\beta\left(1+p_{2}\right) .
\end{aligned}
$$

The single-product monopoly profits $\bar{\Pi}_{1}^{*}$ are attained for $p_{1}=p_{2}$ and $p_{1}=p_{2}+1$. As pointed out above, $p_{1} \geq p_{2}$. Let us again introduce $\delta=p_{1}-p_{2} \in[0,1]$. Then

$$
\begin{aligned}
\bar{\Pi}_{\mathrm{H}_{2}}\left(p_{2}+\delta, p_{2}\right)= & -\frac{p_{2}^{2}}{\hat{W}}+\left(1-\beta-\frac{\delta(1-\delta)}{2 \hat{W}}\right) p_{2} \\
& +\frac{\delta(1-\delta)}{2}\left(1-\frac{\delta}{\hat{W}}\right)-\beta .
\end{aligned}
$$

The first-order condition with respect to $p_{2}$, $\partial p_{2} \bar{\Pi}_{\mathrm{H}_{2}}\left(p_{2}+\delta, p_{2}\right)=0$, yields $p_{2}^{*}(\delta)=\frac{\hat{W}}{2}(1-\beta)-\frac{\delta(1-\delta)}{4}$, and therefore

$$
\begin{aligned}
\bar{\Pi}_{\mathrm{H}_{2}}\left(p_{2}^{*}(\delta)+\delta, p_{2}^{*}(\delta)\right)=\frac{\hat{W}}{4}(1-\beta)^{2}-\beta \\
-\frac{\delta(1-\delta)}{16 \hat{W}}\left(\delta^{2}+7 \delta-4 \hat{W}(1+\beta)\right) \\
=\bar{\Pi}_{1}^{*}+\frac{\delta(1-\delta)}{16 \hat{W}}\left(\delta-\delta_{-}\right)\left(\delta_{+}-\delta\right),
\end{aligned}
$$

where $\delta_{ \pm}=(-7 \pm \sqrt{49+16 \hat{W}(1+\beta)}) / 2$. It is $\delta_{-}<0<\delta_{+}$, and therefore $\bar{\Pi}_{\mathrm{H}_{2}}^{*}$ in expression (31) is greater than $\bar{\Pi}_{\mathrm{H}_{2}}^{*}$, if and only if $0<\delta<\min \left\{\delta_{+}, 1\right\}$. The following lemma helps establishing that the optimal profit $\bar{\Pi}_{\mathrm{H}_{2}}^{*}$ is strictly inferior to $\max \left\{\bar{\Pi}_{\mathrm{H}_{1}}^{*}, \bar{\Pi}_{\mathrm{V}}^{*}\right\}$. In other words, if pure horizontal differentiation is superior to pure vertical differentiation, then separation will maximize profits.

Lemma 2. Let the parameters $0<\beta<1$ and $\hat{W}$ be given. Then for any $\delta \in[0,1]$ :

$$
\delta(1-\delta)\left(\delta-\delta_{-}\right)\left(\delta_{+}-\delta\right)<\max \left\{8 \beta \hat{W},(2 \hat{W}(1-\beta))^{2} / 3\right\} .
$$

Proof. Let $\bar{\delta}=\min \left\{1, \delta_{+}\right\}$. As the RHS of Eq. (32) is always positive, we can limit ourselves to $\delta \in I=[0, \bar{\delta}]$ for which the LHS is nonnegative. Set $f(\delta)$ to be equal to the LHS of Eq. (32). Since $f$ is a polynomial with a set of roots $\left\{\delta_{-}, 0, \delta_{+}, 1\right\}$, where $\delta_{-}<0<\bar{\delta}$, it is

$\max _{\delta \in I}\left|f^{\prime}(\delta)\right|=\max \left\{f^{\prime}(0),-f^{\prime}(\bar{\delta})\right\}$. 
More specifically, by the mean value theorem and the fact that $\left.f^{\prime \prime}\right|_{I}<0$, for all $\delta \in I$ :

$f(\delta) \leq \min \left\{f^{\prime}(0) \cdot \delta,-f^{\prime}(\bar{\delta})(\bar{\delta}-\delta)\right\} \leq \frac{f^{\prime}(\bar{\delta}) f^{\prime}(0) \cdot \bar{\delta}}{f^{\prime}(0)-f^{\prime}(\bar{\delta})}$.

Thereby $f^{\prime}(0)=-\delta_{-} \delta_{+}$and $-f^{\prime}(\bar{\delta})=\max \left\{\delta_{+}\left(\delta_{+}-\delta_{-}\right)\right.$ $\left.\left(1-\delta_{+}\right),\left(1-\delta_{-}\right)\left(\delta_{+}-1\right)\right\}$. Let us first consider the case when $\bar{\delta}=1$, or equivalently

$\hat{W}(1+\beta) \geq 2$.

Then by Eq. (33)

$$
\begin{aligned}
f(\delta) & \leq \frac{-\delta_{-} \delta_{+}\left(1-\delta_{-}\right)\left(\delta_{+}-1\right)}{-\delta_{-} \delta_{+}+\left(1-\delta_{-}\right)\left(\delta_{+}-1\right)} \\
& =\frac{16 \hat{W}(1+\beta)(\hat{W}(1+\beta)-2)}{4 \hat{W}(1+\beta)+4(\hat{W}(1+\beta)-2)} \\
& =\frac{2 \hat{W}(1+\beta)(\hat{W}(1+\beta)-2)}{\hat{W}(1+\beta)-1} .
\end{aligned}
$$

One can verify by straightforward manipulations that the RHS of Eq. (35) is less than $8 \beta \hat{W}$ if and only if

$\hat{W}(1-3 \beta)<2 \frac{1-\beta}{1+\beta}$,

which establishes inequality (32) for all

$$
\begin{aligned}
(\beta, \hat{W}) \in D_{1}= & \left\{(\breve{\beta}, \breve{W}): 0<\breve{\beta}<\frac{1}{3}, \breve{W}<\frac{1-\breve{\beta}}{(1+\breve{\beta})(1-3 \breve{\beta})}\right\} \\
& \cup([1 / 3,1) \times(0, \infty)) .
\end{aligned}
$$

On the other hand, one can verify that the RHS of Eq. (35) is less than $\left(2 \hat{W}(1-\beta)^{2} / 3\right.$ if and only if

$$
\frac{2}{1+\beta}+\frac{2}{3} \frac{(1-\beta)^{2}}{1+\beta} \hat{W}^{2}-\left(\frac{2}{3}\left(\frac{1-\beta}{1+\beta}\right)^{2}+1\right) \hat{W}>0 \text {. }
$$

Restricting $\beta$ to the interval $(0,1 / 3]$, inequality (36) is satisfied if

$$
\begin{aligned}
& 3+(1-\beta)^{2} \hat{W}^{2}-\left(\frac{(1-\beta)^{2}}{1+\beta}+\frac{3}{2}(1+\beta)\right) \hat{W} \\
& \geq 3+\left[\frac{2}{3}(1-\beta) \hat{W}-\left(\frac{(1-\beta)^{2}}{1+\beta}+\frac{3}{2}(1+\beta)\right)\right] \hat{W}>0 .
\end{aligned}
$$

The expression in square brackets can be further minorized by setting $\beta=1 / 3$, based on the fact that the derivative of that expression with respect to $\beta$,

$-\frac{4}{3} \frac{1}{1+\beta}-\frac{4}{3} \frac{1-\beta}{(1+\beta)^{2}}+2 \frac{1-\beta}{1+\beta}+\frac{(1-\beta)^{2}}{(1+\beta)^{2}}-\frac{3}{2}$

is negative on $(0,1 / 3]$. In particular,

$$
\begin{aligned}
3+ & \hat{W}\left[\frac{2}{3}(1-\beta) \hat{W}-\left(\frac{(1-\beta)^{2}}{1+\beta}+\frac{3}{2}(1+\beta)\right)\right]_{\beta=1 / 3} \\
& =\frac{1}{9}(\hat{W}-3)(4 \hat{W}-9)>0
\end{aligned}
$$

implies Eq. (32) for all $(\beta, \hat{W}) \in D_{2}=(0,1 / 3] \times((0,9 / 4)$ $\cup(3, \infty))$. Alternately one can minorize the RHS of Eq. (36) by substituting

$$
\hat{W}=\frac{2(1-\beta)^{2}+3(1+\beta)^{2}}{4(1-\beta)^{2}(1+\beta)},
$$

its unique minimizer. Relation (36) is satisfied for a given $\beta \in(0,1 / 3)$ and all $\hat{W}>0$ if

$23 \beta^{4}-20 \beta^{3}-150 \beta^{2}-20 \beta+23>0$.

The last polynomial has the set of approximate roots $\{-2.0221,-.4945, .3269,3.0593\}$, so that Eq. (32) follows for $(\beta, \hat{W}) \in D_{3}=(0,3 / 10) \times(0, \infty)$. Since $D_{1} \cup D_{2} \cup D_{3}=$ $(0,1) \times(0, \infty)$, we have indeed shown that Eq. (32) holds for all relevant ${ }^{22}(\beta, \hat{W})$ whenever $\bar{\delta}=1$. Let us now examine the case when $\bar{\delta}=\delta_{+}<1$ or in other words

$0<\hat{W}<\frac{2}{1+\beta}$.

Using Eq. (33) write

$$
\begin{aligned}
f(\delta) & \leq \frac{-\delta_{-} \delta_{+}^{3}\left(\delta_{+}-\delta_{-}\right)\left(1-\delta_{+}\right)}{-\delta_{-} \delta_{+}+\delta_{+}\left(\delta_{+}-\delta_{-}\right)\left(1-\delta_{+}\right)} \\
& <\frac{\delta_{+}^{2}\left(\delta_{+}-\delta_{-}\right)\left(1-\delta_{+}\right)}{2-\delta_{+}} \\
& =\frac{1}{4} \frac{\sqrt{49+16 \eta}(-9+\sqrt{49+16 \eta})(-7+\sqrt{49+16 \eta})^{2}}{-11+\sqrt{49+16 \eta}},
\end{aligned}
$$

where $\eta=\hat{W}(1+\beta)$ and by Eq. (37) it is $0<\eta<2$. Straightforward computations yield that expression (38) is less than $8 \beta \hat{W}=8 \eta(1-1 /(1+\beta))$ if and only if

$$
(\beta, \eta) \in E_{1}=\left\{(\breve{\beta}, \breve{\eta}): 0<\breve{\beta}<R_{1}(\breve{\eta}), 0<\breve{\eta}<2\right\},
$$

\footnotetext{
$22 \bar{\delta}=1$ is equivalent to Eq. (34), which slightly restricts the relevant domain of $(\beta, \hat{W})$ to a subset of $(0,1) \times(0, \infty)$.
} 
where

$$
\begin{aligned}
R_{1}(\eta)= & \left(2 \eta^{3}-153 \eta^{2}-537 \eta-294\right)^{-1}\left(2 \eta^{3}+279 \eta^{2}\right. \\
& +306 \eta-1764+\sqrt{49+16 \eta}\left(15 \eta+12 \eta^{2}\right. \\
& -294)+\left[4032 \eta^{5}+70,620 \eta^{4}-91,110 \eta^{3}\right. \\
& -1,744,848 \eta^{2}-444,528 \eta+7,260,624 \\
& +\sqrt{49+16 \eta}\left(48 \eta^{5}+6756 \eta^{4}+14,538 \eta^{3}\right. \\
& \left.\left.\left.-197,208 \eta^{2}-232,848 \eta+1,037,232\right)\right]^{1 / 2}\right) .
\end{aligned}
$$

On the other hand, expression (38) is less than $(2 \hat{W}(1+$ $\beta))^{2} / 3=4 \eta^{2}\left(1-4 /(1+\beta)+4 /(1+\beta)^{2} / 3\right.$ if and only if

$$
(\beta, \eta) \in E_{2}=\left\{(\breve{\beta}, \breve{\eta}): R_{2}(\breve{\eta})<\breve{\beta}<1,0<\breve{\eta}<2\right\},
$$

where

$R_{2}(\eta)=\frac{\sqrt{49+16 \eta}(23 \eta+98)-16 \eta^{2}-273 \eta-686}{16 \eta^{2}+295 \eta+686-\sqrt{49+16 \eta}(25 \eta+98)}$.

Since $R_{1}(\eta)>R_{2}(\eta)$ for all $0<\eta<2$, it is $E_{1} \cup E_{2}=(0$, $1) \times(0,2)$, and therefore Eq. (32) holds also for the case that $\bar{\delta}=\delta_{+}$, which concludes the proof of Lemma 2 .

As mentioned before, based on Lemma 2 as well as Eqs. (10), (29), and (31) pure horizontal differentiation in the overlap mode is strictly dominated by either pure vertical differentiation or pure horizontal differentiation in the separation mode. This concludes the proof of Proposition 3.

Proof of Proposition 4. (i) For zero versioning costs, condition (19) is necessary and sufficient, since the viability domains of single-product monopoly, pure vertical differentiation, and mixed differentiation are subsets of the region of positive profits for pure horizontal differentiation in separation mode, given precisely by Eq. (19). Thus, if the latter condition is satisfied there exists $\varepsilon>0$ such that $\alpha_{H} \in[0, \varepsilon]$ is small enough for the resulting profits to be positive. (ii) This is an immediate consequence of Eq. (19) being necessary and sufficient for viability under zero versioning costs. (iii) From $\hat{W} \in\left(2 \beta /(1-\beta)^{2}, 4 \beta /(1-\beta)^{2}\right)$ it follows that a singleproduct monopoly is not viable according to Eq. (7), while at the same time Eq. (19) is satisfied.

\section{Details on the horizontal-overlap case under delayed} differentiation. Since $q_{1} \geq q_{2}$, we have that necessarily $p_{1} \geq p_{2}$, which generally implies price dispersion for goods of equal quality. In the following it is useful to distinguish three cases. (i) If $q_{1}-p_{2}<1$, then $q_{1}-p_{1}<1$ and $q_{2}-p_{2}<1$. We can conclude that $p_{1}, p_{2} \in\left[q_{1}-1\right.$, $\left.q_{1}\right]$ or in other words that $p_{1}-p_{2}<1$. Thus, $q_{1}=q_{2}$ (pure horizontal differentiation). The profit-maximization problem is

$$
\begin{aligned}
\left(p_{1}, p_{2}\right)= & \arg \max _{p_{1}, p_{2}}\left\{\frac{p_{1}\left(W-p_{1}\right)\left(1-p_{1}+p_{2}\right)}{2 W}\right. \\
& \left.+\frac{p_{2}\left(p_{1}-p_{2}\right)\left(q_{1}-p_{2}\right)}{W}+\frac{p_{2}\left(W-p_{1}\right)\left(1+p_{1}-p_{2}\right)}{2 W}\right\} \\
= & \arg \max _{p_{1}, p_{2}}\left\{\frac{W-p_{1}}{2 W}\left(p_{1}+p_{2}-\left(p_{1}-p_{2}\right)^{2}\right)\right. \\
& \left.+\frac{p_{2}\left(p_{1}-p_{2}\right)\left(q_{1}-p_{2}\right)}{W}\right\} .
\end{aligned}
$$

Setting $\delta=p_{1}-p_{2} \in[0,1]$ it is possible to rewrite the profit function as

$\Pi_{\mathrm{H}_{2}}\left(p_{2}+\delta, \delta\right)=\frac{\left(W-p_{2}-\delta\right)\left(2 p_{2}-\delta(1-\delta)\right)+2 p_{2} \delta\left(q_{1}-p_{2}\right)}{2 W}-\beta q_{1}$.

Any interior extremum satisfies the necessary optimality condition for $p_{2}$, from which we obtain

$p_{2}^{*}(\delta)=\frac{2 W+\delta\left(2\left(q_{1}-1\right)-(1-\delta)\right)}{4(1+\delta)}$.

By substituting this expression, the profit function $\Pi_{\mathrm{H}_{2}}$ can be expressed as a function of $\delta$ only, and the firstorder condition with respect to $\delta$ is

$a \delta^{4}+b \delta^{3}+c \delta^{2}+d \delta+e=0$,

with $a=27 / 16, b=\left(q_{1}+3\right) / 2-W, c=\left(q_{1}^{2}-5 W\right) / 4-17 /$ $16, d=\left(q_{1}^{2}-3 q_{1}+W\right) / 2+1 / 8$, and $e=-W\left(W+1-2 q_{1}\right) /$ 4. To analytically find the roots of this fourth-order polynomial we follow Ref. [7]. Substituting $y=\delta+b /$ (4a), Eq. (42) can be written equivalently in the reduced form

$y^{4}+P y^{2}+Q y+R=0$,

where $P=(c / a)-3 b^{2} / 8 a^{2}, Q=d / a-c b / a^{2}+b^{3} / 8 a^{3}$, and $R=e / a+\left(c b^{2} / 16-d b / 4\right) / a^{2}-3 b^{4} / 256 a^{4}$. The solution behavior of Eq. (43) depends on the behavior of its resolvent cubic,

$z^{3}+2 P z^{2}+\left(P^{2}-4 R\right) z-Q^{2}$

the roots of which are all real ${ }^{23}$ and given by $z_{k}=2 \rho \cos (\varphi /$ $3+2(k-1) \pi / 3)$, for $k=1,2,3$, with $\rho=\sqrt{4 P^{2}-3 Q} / 3$ and $\varphi=\arccos \left(\left(2 P\left(P^{2}-4 R\right) / 3+Q^{2}-16 P^{3} / 27\right) /\left(2 \rho^{3}\right)\right)$.

\footnotetext{
${ }^{23}$ This follows from the fact that the discriminant $\Delta=\left(P^{2}-4 R-4 P^{2}\right)$ $3) / 3)^{3}+\left(16 P^{3} / 27-2 P\left(P^{2}-4 R\right) / 3-Q^{2}\right)^{2} / 4$ is negative in the relevant domain, which can be verified numerically.
} 
From this, the roots of the reduced-form polynomial in Eq. (43) can be computed to

$$
\begin{aligned}
& y_{1}=\left(\sqrt{z_{1}}+\sqrt{z_{2}}-\sqrt{z_{3}}\right) / 2, \\
& y_{2}=\left(\sqrt{z_{1}}-\sqrt{z_{2}}+\sqrt{z_{3}}\right) / 2, \\
& y_{3}=\left(\sqrt{z_{1}}+\sqrt{z_{2}}+\sqrt{z_{3}}\right) / 2, \\
& y_{4}=\left(\sqrt{z_{1}}-\sqrt{z_{2}}-\sqrt{z_{3}}\right) / 2,
\end{aligned}
$$

and a local maximizer of $\Pi_{\mathrm{H}_{2}}\left(p_{2}^{*}(\delta)+\delta, p_{2}^{*}(\delta)\right)$ is

$\delta^{*}=y_{1}-\frac{b}{4 a}$,

as the second-order condition is satisfied at that point. ${ }^{24}$

(ii) If $q_{1}-p_{2} \geq 1$, then the optimization problem is

$$
\begin{aligned}
\left(p_{1}, p_{2}\right)= & \arg \max _{p_{1}, p_{2}}\left\{\frac{p_{1}\left(W-p_{1}\right)\left(1-p_{1}+p_{2}\right)}{2 W}\right. \\
+ & \left.\frac{p_{2}\left(p_{1}-p_{2}\right)}{W}+\frac{p_{2}\left(W-p_{1}\right)\left(1+p_{1}-p_{2}\right)}{2 W}\right\} \\
= & \arg \max _{p_{1}, p_{2}}\left\{\frac{W-p_{1}}{2}\left(p_{1}+p_{2}-\left(p_{1}-p_{2}\right)^{2}\right)\right. \\
& \left.+p_{2}\left(p_{1}-p_{2}\right)\right\} .
\end{aligned}
$$

The quality $q_{2}$ is actually indeterminate in $\left[1+p_{2}, q_{1}\right]$, but without loss of generality we set $q_{2}=q_{1}$ (which is true for even very small positive versioning cost). Note that $p_{1}-p_{2} \leq 1$, otherwise there is only one product. Also, this case is equivalent to the corresponding ex-ante versioning situation for $\beta=0$. Introducing $\delta=p_{1}-p_{2} \in[0,1]$, we obtain

$\Pi_{\mathrm{H}_{2}}\left(p_{2}+\delta, \delta\right)=\frac{\left(W-p_{2}-\delta\right)\left(2 p_{2}+\delta-\delta^{2}\right)+2 p_{2} \delta}{2 W}-\beta q_{1}$.

Assuming that there is an interior extremum the firstorder condition with respect to $p_{2}$ is

$2 W-4 p_{2}-\delta(1-\delta)=0$,

so that $p^{*}(\delta)=W / 2-\delta(1-\delta) / 4$. The resulting expression for the profits under horizontal versioning with overlap is

$\Pi_{\mathrm{H}_{2}}(\delta)=\frac{W}{4}-\beta q_{1}+\frac{\delta(1-\delta)\left(\delta-\delta_{-}\right)\left(\delta_{+}-\delta\right)}{16 W}$,

where $\delta_{ \pm}=(-7 \pm \sqrt{16 W+49}) / 2$. The first-order condition with respect to $\delta$ is then

$\delta^{3}+\frac{9}{2} \delta^{2}-\left(2 W+\frac{7}{2}\right) \delta+W=0$.

Using the cardanic formula to solve the cubic equation (47) it turns out that for any $W$ there are three real roots,

\footnotetext{
${ }^{24}$ An analytical test of the second-order condition is complicated, but it can be readily verified numerically.
}

of which only one is associated with an interior maximum of the fourth-order polynomial at Eq. (46),

$\delta^{*}(W)=\sqrt{\frac{8 W+41}{3}} \sin \left(\frac{\pi}{6}-\frac{1}{3} \arccos \left(\frac{48(W+3)}{\sqrt{(8 W+41)^{3} / 3}}\right)\right)-\frac{3}{2}$.

It is $\delta^{*}(W) \in[0,1 / 2]$ strictly increasing in $W$ with $\delta^{*}(0)=0$ and $\lim _{W \rightarrow \infty} \delta^{*}(W)=1 / 2$.

(iii) In the intermediary case with binding constraint, $q_{1}-p_{2}=1$, the optimization problem is identical to (45), subject to $p_{2}=q_{1}-1$,

$$
\begin{aligned}
\Pi_{\mathrm{H}_{2}}(\delta)= & \frac{\left(W-\left(q_{1}-1\right)\right)\left(q_{1}-1\right)}{W}-\beta q_{1} \\
& +\frac{\delta(1-\delta)(A-\delta)}{2 W}
\end{aligned}
$$

with the abbreviation $A=W-\left(q_{1}-1\right)$. The first-order necessary optimality condition is $3 \delta^{2}-2(A+1) \delta+A=0$, and the maximizer is therefore

$\delta^{*}=\frac{A+1-\sqrt{A^{2}-A+1}}{3}$.

The second-order condition is satisfied here, since $\Pi_{\mathrm{H} 2}^{\prime \prime}\left(\delta^{*}\right)=-2 \sqrt{A^{2}-A+1}<0$. This concludes our $\mathrm{H}_{2}$ analytical discussion of the horizontal-overlap case under delayed differentiation.

Proof of Proposition 5. (i) It is sufficient to show that the profit function under mixed ex-post differentiation is dominated by pure horizontal differentiation with overlap. Let $\left(p_{1}, q_{1}\right)$ be such that $1 / 2 \leq q_{1}-p_{1}<1$. Under horizontal separation (in which the local mixeddifferentiation optimum occurs) this implies that $q_{1}-$ $p_{1}=1-\left(q_{2}-p_{2}\right)<1$, and furthermore, as shown in Section 4.1 , then $p_{1}^{*}=p_{2}^{*}=W / 2$. Thus, also $q_{1}-p_{2}^{*}<1$ so that we can compare the profit function under horizontal separation to the maximand in Eq. (41) discussed earlier in this Appendix for the case that $q_{1}-$ $p_{2}<1$. Subtracting the latter from the former yields $-p_{1}$ $\left(W-p_{1}\right)\left(1-\left(q_{1}-p_{2}\right)\right) / 2-p_{2}\left(W-p_{2}\right)\left(q_{1}-p_{2}\right)-p_{2}\left(p_{1}-\right.$ $\left.p_{2}\right)\left(q_{1}-p_{2}\right)\left(q_{1}-p_{2}\right)<0$, so that mixed differentiation is strictly dominated. (ii) The claim that horizontal cannibalization is sometimes optimal can be shown by example and thus Fig. 7 is sufficient as a proof. It is not optimal for large $q_{1}$ (compared to $W$ ), since it can be shown analogously to the computation in part (i) that pure vertical differentiation is globally better. 
For $q_{1}$ small compared to $W$ horizontal overlap cannot be achieved. Thus, horizontal overlap can only be best for "intermediate" realizations of $W$. This concludes the Proof of Proposition 5.

Proof of Proposition 6. Assume first that ex post pure vertical differentiation is optimal, independent of the demand realization $W$. Then differentiating the expression $(22)$ for $\Pi_{\mathrm{V}}^{*}\left(q_{1} ; W\right)$ and then taking the expectation with respect to $W$ yields ${ }^{25}$

$$
\begin{aligned}
-\beta & +\int_{W_{1}}^{W_{2}}\left(1-\frac{3\left(q_{1}^{* *}-1\right)}{2 w}\right) f(w) \mathrm{d} w \\
& +\int_{W_{2}}^{\bar{s}} p_{1}^{*}\left(1-\frac{3 p_{1}^{*}}{4 w}\right) f(w) \mathrm{d} w=0,
\end{aligned}
$$

where $W_{1}, W_{2}, p_{1}^{*}$, and $\bar{s}$ are as the corresponding part of Proposition 6. The last equation is equivalent to Eq. (25). Next assume that ex post pure vertical differentiation is optimal, independent of the demand realization $W$. As before we determine the first-order condition by first taking the derivative of $\Pi_{\mathrm{H}_{1}}\left(q_{1} ; W\right)$ in Eq. (24) with respect to $q_{1}$ and then taking the expectation with respect to $W$. This yields

$$
\begin{aligned}
-\beta & +\int_{W_{1}}^{W_{2}}\left(1-\frac{2 q_{1}^{* *}-1}{w}\right) f(w) \mathrm{d} w \\
& +2 \int_{W_{2}}^{\bar{s}} p_{1}^{*}\left(1-\frac{p_{1}^{*}}{w}\right) f(w) \mathrm{d} w=0,
\end{aligned}
$$

which is equivalent to Eq. (26) using the definitions of $W_{1}, W_{2}, p_{1}^{* *}$, and $\bar{s}$ as in the corresponding part of Proposition 6.

Proof of Proposition 7. (ii) By Berge's maximum theorem [5] the option value $V_{\mathrm{d}}$ is continuous in $\beta$ as difference of two continuous functions $\bar{\Pi}^{* *}$ and $\bar{\Pi}^{*}$. Also as a consequence of the maximum theorem both maximizers $q_{1}^{* *}$ and $q_{1}^{*}$ are generally set-valued and upper semicontinuous in the parameter $\beta$. The maximizers are set-valued if and only if the firm is indifferent between several different $q_{1}$-values. Let $\hat{W}>0$ be given. Following our developments in Section $3, q_{1}^{*}(\beta)$, $0 \leq \beta \leq 1$, is given by

$$
q_{1}^{*}(\beta)= \begin{cases}1+2 \hat{W}(1-\beta) / 3, & \text { if } \beta \geq \beta^{\prime}, \\ 1 / 2+\hat{W}(1-\beta) / 2, & \text { if } \beta \in\left[\beta^{\prime}, \beta^{\prime \prime}\right], \\ 0, & \text { otherwise, }\end{cases}
$$

\footnotetext{
${ }^{25}$ This means to switch differentiation and integration when determining the first-order conditions of the optimization problem (20). This naturally presupposes sufficient regularity of the probability density function $f$.
}

where $\beta^{\prime}=(3+\hat{W}-\sqrt{9+6 \hat{W}}) / \hat{W}$ and $\beta^{\prime \prime}=(1+\hat{W}-$ $\sqrt{1+2 \hat{W}}) / \hat{W}$. Only for $\beta \in\left\{\beta^{\prime}, \beta^{\prime \prime}\right\}$ is $q_{1}^{*}$ possibly set-valued; on $\left[0, \beta^{\prime \prime}\right) \backslash\left\{\beta^{\prime}\right\}$ it is strictly monotonically decreasing. Similarly, the optimal flagship quality under delayed differentiation, $q_{1}^{* *}$, can only be set-valued at $0<\beta_{1}, \beta_{2}, \beta_{3}<1$, where $\beta_{1} \leq \beta^{\prime}<\beta_{2}<\beta^{\prime \prime} \leq \beta_{3}$, corresponding to the vertical-contingent $\left(\beta_{1}\right)$, contingenthorizontal $\left(\beta_{2}\right)$, and horizontal-no entry $\left(\beta_{3}\right)$ mode transitions. We have noted in Section 4 that expressions (22) and (24) are continuously differentiable in $q_{1}$. The same is true for the corresponding expression in the overlap mode. In fact, these expressions are twice continuously differentiable ("smooth") almost everywhere, and thus the envelope theorem can be applied in the smooth portions, so that we obtain relation (27). (i) At $\beta=\beta^{\prime}$ the firm is indifferent between horizontal and vertical versioning modes. But if the firm can observe demand $W=\hat{W}$ before taking the versioning decision, ${ }^{26}$ then it generally will not be indifferent between horizontal and vertical differentiation, so that a state-contingent policy of intermediate ex-ante quality will at least weakly increase profits at $\beta=\beta^{\prime}$. As a consequence, $q_{1}^{* *}\left(\beta^{\prime}\right) \in\left(q_{1}^{*}\left(\beta^{\prime}+\right), q_{1}^{*}\left(\beta^{\prime}-\right)\right)$ so that with Eq. (27) we obtain a local maximum of $V_{\mathrm{d}}$ at $\beta=\beta^{\prime}$, in other words a state-contingent policy will generally improve profits around $\beta^{\prime}$. Consider now $\beta=0$. Then $q_{1}^{* *}(0)=1+2 \bar{s} / 3$ with $\bar{s}=\max \{\mathcal{S}\}$. On the other hand, it is following Section 3.2: $q_{1}^{*}(0)=1+2 \hat{W} / 3 \leq q_{1}^{* *}(0)$ because generally $\bar{s} \geq \hat{W}$. (iii) This part is trivial: for $\beta \geq \beta_{3}, \beta^{\prime \prime}$, the firm does not enter the market and thus the option of being able to delay differentiation is not worth anything.

Proof of Proposition 8. (i) Consider first the case of pure vertical differentiation. Consumer surplus $\mathrm{CS}_{\mathrm{V}}$ is then

$$
\begin{aligned}
\mathrm{CS}_{\mathrm{V}} & =\frac{1}{W} \int_{0}^{\min \left\{u_{1}, 1\right\}}\left[\left(u_{1}-v\right)\left(w-p_{1}\right)+\left(u_{1}-v\right)\right] \mathrm{d} v \\
& =\frac{W-p_{2}}{W}\left[u_{1} v-\frac{v^{2}}{2}\right]_{0}^{\min \left\{u_{1}, 1\right\}},
\end{aligned}
$$

an expression equivalent to the first entry in Table 1. The consumer surplus for pure horizontal differentiation with separation follows in the same manner. We

\footnotetext{
${ }^{26}$ If $W=\hat{W}$ (which can only happen with positive probability for certain "pathological" discrete distributions of $W$ ), then the firm might be indifferent between horizontal and vertical versioning modes, even under delayed differentiation.
} 
now turn to the case to horizontal differentiation with overlap, for which the consumer surplus can be computed as follows:

$$
\begin{aligned}
\mathrm{CS}_{\mathrm{H}_{2}}= & \frac{W-p_{1}}{W}+\frac{W-p_{2}}{W} \int_{0}^{\frac{1+p_{1}-p_{2}}{2}}\left(u_{2}-v\right) \mathrm{d} v \\
& +\frac{p_{1}-p_{2}}{W} \int_{0}^{\min \left\{u_{1}, 1\right\}}\left(u_{2}-v\right) \mathrm{d} v .
\end{aligned}
$$

Using the abbreviation $\delta=p_{1}-p_{2}$, we obtain the third expression in Table 1 by straightforward integration. (ii) Substituting the ex-ante optimal product portfolio determined in Section 3 into the first two expressions for consumer surplus in Table 1, one immediately obtains Eq. (28).

\section{References}

[1] K.S. Anand, H. Mendelson, Postponement and information in a supply chain, Working Paper, Kellogg Graduate School of Management, Northwestern University, 1998.

[2] S. Anderson, A. de Palma, J.-F. Thisse, Discrete-Choice Theory of Product Differentiation, MIT Press, Cambridge, MA, 1992.

[3] Y. Bakos, E. Brynjolfsson, Bundling information goods: pricing, profits, and efficiency, Management Science 45 (12) (1999) $1613-1630$.

[4] J. Beath, Y. Katsoulacos, The Economic Theory of Product Differentiation, Cambridge University Press, New York, NY, 1991.

[5] C. Berge, Espaces Topologiques et Fonctions Multivoques, Dunod, Paris, France, 19591.

[6] H.K. Bhargava, V. Choudhary, Information goods and vertical differentiation, Journal of Management Information Systems 18 (2) (2001) 89-106.

[7] I.N. Bronshtein, K.A. Semendyayev, Handbook of Mathematics, Springer, New York, NY, 1997.

[8] E. Chamberlin, The Theory of Monopolistic Competition, Harvard University Press, Cambridge, MA, 1933.

[9] J.M. Conrad, Quasi-option value and the expected value of information, Quarterly Journal of Economics 94 (4) (1980) 813-820.

[10] H. Cremer, J.-F. Thisse, Location models of horizontal differentiation: a special case of vertical differentiation models, Journal of Industrial Economics 39 (4) (1991) 383-390.

[11] R.J. Deneckere, R.P. McAfee, Damaged goods, Journal of Economics and Management Strategy 5 (2) (1996) 149-174.

[12] M. Fisher, K. Ramdas, K.T. Ulrich, Component sharing in the management of product variety: a study of automotive braking systems, Management Science 45 (3) (1999) 297-315.

[13] X. Geng, M.B. Stinchcombe, A.B. Whinston, Bundling information goods of decreasing value, Management Science 51 (4) (2005) 662-667.

[14] W.M. Gorman, Conditions for additive separability, Econometrica 36 (3-4) (1968) 605-609.

[15] H. Hotelling, Stability in competition, Economic Journal 39 (153) (1929) 41-57.
[16] A. Huchzermeier, C.H. Loch, Project management under risk: using the real options approach to evaluate flexibility in $R \& D$, Management Science 47 (1) (2001) 85-101.

[17] R. Jones, H. Mendelson, Product and price competition for information goods, Working Paper, Graduate School of Business, Stanford University, 1998.

[18] V. Krishnan, K.T. Ulrich, Product development decisions: a review of the literature, Management Science 47 (1) (2001) $1-21$.

[19] J.-J. Laffont, E. Maskin, J.-C. Rochet, Optimal nonlinear pricing with two-dimensional characteristics, in: T. Groves, R. Radner, S. Steiger (Eds.), Information, Incentives, and Economic Mechanisms: Essays in Honor of Leonid Hurwicz, University of Minnesota Press, Minneapolis, Minnesota, 1987, pp. 255-266.

[20] K.J. Lancaster, A new approach to consumer theory, Journal of Political Economy 74 (2) (1966) 132-157.

[21] H.L. Lee, C.S. Tang, Modeling the costs and benefits of delayed product differentiation, Management Science 43 (1) (1997) 40-53.

[22] R. McDonald, D. Siegel, The value of waiting to invest, Quarterly Journal of Economics 101 (4) (1986) 707-728.

[23] J.A. Mirrlees, An exploration in the theory of optimal income taxation, Review of Economic Studies 38 (2) (1971) 175-208.

[24] K.S. Moorthy, I. Png, Market segmentation, cannibalization, and the timing of product introductions, Management Science 38 (3) (1992) 345-359.

[25] M. Mussa, S. Rosen, Monopoly and product quality, Journal of Economic Theory 18 (2) (1978) 301-317.

[26] J.C. Panzar, R.D. Willig, Economies of scope, American Economic Review 71 (2) (1981) 268-272.

[27] J. Perloff, S. Salop, Equilibrium with product differentiation, Review of Economic Studies 52 (1) (1985) 107-120.

[28] R.S. Pindyck, Irreversible investment, capacity choice, and the value of the firm, American Economic Review 78 (5) (1988) 969-985.

[29] J.-C. Rochet, P. Choné, Ironing, sweeping and multidimensional screening, Econometrica 66 (4) (1998) 783-826.

[30] S. Salop, Monopolistic competition with outside goods, Bell Journal of Economics 10 (1) (1979) 141-156.

[31] A. Shaked, J. Sutton, Product differentiation and industrial structure, Journal of Industrial Economics 36 (2) (1987) 131-146.

[32] C. Shapiro, H. Varian, Versioning: the smart way to sell information, Harvard Business Review 76 (6) (1998) 107-114.

[33] J.M. Swaminathan, S.R. Tayur, Managing broader product lines through delayed differentiation using vanilla boxes, Management Science 44 (12-2) (1998) S161-S172.

[34] J. Tirole, The Theory of Industrial Organization, MIT Press, Cambridge, MA, 1988.

[35] K.T. Ulrich, The role of product architecture in the manufacturing firm, Research Policy 24 (3) (1995) 419-440.

[36] J.A. Van Mieghem, Investment strategies for flexible resources, Management Science 44 (8) (1998) 1071-1078.

[37] T.A. Weber, Mixed versioning of information goods under incomplete information, Proceedings of the 22nd International Conference on Information Systems, New Orleans, LA, 2001, pp. 81-83.

[38] B. Weisbrod, Collective-consumption services of individual consumption goods, Quarterly Journal of Economics 78 (3) (1964) 471-477. 


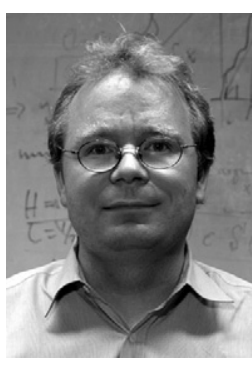

Thomas A. Weber is Assistant Professor of Management Science and Engineering at Stanford University. His research is concerned with managerial decision-making and the economics of information systems. Weber received his doctorate in Managerial Science and Applied Economics from the Wharton School of the University of Pennsylvania in 2003. From 1998 to 2002 he was senior consultant with the Boston Consulting Group. $\mathrm{He}$ holds engineering degrees from École Centrale Paris and Technical University Aachen, and received Master of Science degrees in Electrical Engineering and Computer Science as well as in Technology and Policy from the Massachusetts Institute of Technology. In 2003 he was named a David T. Morgenthaler II Faculty Scholar at Stanford University. He presently serves as an associate editor for Management Science. 